\title{
Collisions between protoplanets: data analysis and classification
}

\author{
Akiyoshi Nouda ${ }^{1}$, Hiroyuki Emori ${ }^{2}$, and Kiyoshi Nakazawa ${ }^{1}$ \\ ${ }^{1}$ Department of Earth and Planetary Sciences, Tokyo Institute of Technology, Tokyo 152-8551, Japan \\ ${ }^{2}$ Department of Business Management, Shumei-Univ., Chiba 276-0003, Japan
}

(Received September 25, 2006; Revised July 8, 2008; Accepted November 24, 2008; Online published July 27, 2009)

\begin{abstract}
Collisions between protoplanets are common events in the later stage of the planetary formation process. Protoplanets grew into present-day planets through these collisional events. It is therefore necessary to obtain information about redistribution of mass and angular momentum between colliding two bodies in order to study the growth rates of planets and the origin of their spin. We have started a series of systematic studies on this theme, and this is our first report. Here, we concentrate on the impact between one protoplanet, which has one Earth mass, and another protoplanet with 0.06 Earth mass. The motion of two bodies are pursued numerically by the SPH simulation method. We change the minimum distance between the two bodies as the variable parameter to specify their orbital angular momentum. For each value of the parameter, we repeat the numerical simulations and obtain final states of bodies. Such various final states are examined by criteria developed to distinguish some clusters formed by many SPH particles. We determine instantaneous orbital elements for relative motion between central protoplanet and each cluster. From this information, we forecast the destiny of each cluster, for example, to be merged into the target body or trapped. Then we determine the initial conditions that result in final characteristic states. The 5 states are the merging state in which the impactor is absorbed to the target, the multiple impact state in which the impactor collides with the target again and again, the disruption escaping state in which the impactor is tidally disrupted and a large part of the impactor escapes from the target, the trapping state in which the impactor is trapped by the target, and the passing state in which the impactor passes through as it is.
\end{abstract}

Key words: Collisional product, planetary formation, giant impacts, collisional simulation, SPH.

\section{Introduction}

It has been widely accepted that the formation of the planets proceeded with successive, long-term collisional events between planetesimals and protoplanets. Hence, it is essentially governed by collisional probabilities among those gravitating bodies.

In the pioneering studies on planetary formation, the growth of planetesimals and protoplanets is described by the statistical manner in which the time evolution of the mass distribution function of planetesimals is pursued (e.g., Safronov and Zvjagina, 1969; Hayashi et al., 1985). In these studies, it is assumed, for the sake of simplicity, that the collisional cross section is given by the Safronov formula and that two planetesimals coalesce completely without a loss of their masses after they collide with each other. The Safronov formula is readily deduced from the conservation laws of the energy and the angular momentum of the two bodies, and is,

$$
\sigma=\pi\left(r_{1}+r_{2}\right)^{2}\left\{1+\frac{G\left(m_{1}+m_{2}\right)}{v^{2}\left(r_{1}+r_{2}\right)}\right\},
$$

where $r_{i}$ and $m_{i}$ are the radius and the mass of the collisional bodies, respectively, and $v$ is the relative velocity when the distance between the two bodies is infinite.

Copyright (c) The Society of Geomagnetism and Earth, Planetary and Space Sciences (SGEPSS); The Seismological Society of Japan; The Volcanological Society of Japan; The Geodetic Society of Japan; The Japanese Society for Planetary Sciences; TERRAPUB
Remarkable developments in computer architecture has made it possible to introduce more realistic expressions into the study of the planetary formation. The collisional cross section given in Eq. (1) has been replaced by a three-body formula evaluated from a number of the orbital calculations of the two celestial bodies rotating around the Sun (e.g., Nakazawa et al., 1989a, b; Ida and Nakazawa, 1989; Lissauer et al., 1997). The multiple growth processes of planetesimals are also directly pursued by means of the powerful $N$-body simulation code (e.g., Ida and Makino, 1992a, b; Kokubo and Ida, 1996, 1998). However, it should be noted that, even in these new studies, the coalescent probability is determined by the geometrical cross section between rigid bodies-even though it is readily conjectured that the collision between two bodies does not always lead to their complete coalescence. In some cases, the target protoplanet may lose its mass as a result of the shallow collision of a planetesimal. In other cases, even without any direct collision, a protoplanet may absorb the mass of a planetesimal through the tidal fragmentation. Although a number of previous studies on planetary formation have taken into account the varieties of collisional products on the basis of the laboratory experiments, practical knowledge on the collisional products between celestial-scale colliding bodies is still inadequate. For example, Agnor and Asphaug (2004) studied the collisions between two celestial objects by changing the impact velocity and the impact angle. They pursued collisions between two $0.10 M_{\oplus}$ planetary embryos and con- 
cluded that more than half of collisions between like-sized planetary embryos do not result in accumulation. Though their results are reliable, the masses of the colliding bodies are limited to the case of the Martian mass and the range of the adopted impact parameter is not wide enough to detect a general behavior pattern of the collisional products, such as the redistribution of the mass and the angular momentum.

Another important problem, the origin of the Moon, has been studied by many authors in terms of a giant impact (Hartmann and Davis, 1975; Cameron and Ward, 1976; Benz et al., 1986, 1987; see review by Wood, 1986). Cameron, Benz, and co-workers have extensively researched the giant impacts using numerical simulations. Benz et al. $(1986,1987)$ carried out pioneering works that showed possibilities to form the Moon directly by a single impact. However, it was revealed thereafter impacts with low velocities and large angular momenta, in other words, "grazing" impacts, would be needed to form the Moon directly. In the 1990s, based on more precise numerical simulations with more realistic equations of state, it was recognized that the chance that the Moon was formed directly by single collision was small. Alternatively, Cameron and his co-workers asserted that Moon was accumulated from a disk around the Earth, which was formed by the giant impact (Benz et al., 1989; Cameron and Benz, 1991; Cameron, 1997). According to their studies, however, in order to scatter the materials of the lunar mass beyond the Roche limit by a single impact, the colliding system must have a large angular momentum that is about twice as much as that of the present Earth-Moon system. Focusing on this difficulty, Ida et al. (1997) investigated the diffusion process of the disk inside the Roche limit by $N$-body simulations and found that the materials of the lunar mass are readily transferred outside the Roche limit when the disk mass is several times larger than the lunar mass. More precise simulations about potential Moon generating impacts were subsequently made by many authors (Cameron, 2000; Canup and Asphaug, 2001; Canup et al., 2001; Canup, 2004b; review by Canup, 2004a; Wada et al., 2006). After Canup (2004a), the impactor-target ratio is limited in a rather narrow range $(0.11 \sim 0.14)$ for forming the Moon. In almost all of the studies mentioned above, reseachers focused on the formation of the Moon. Consequently, we cannot find any explanation of the general behavior of the collisional phenomena from these studies.

In terms of planetary formation, tidal disruption is also an important process during the growth of planetesimals and protoplanets. Tidal disruption is a classical problem in astrophysics and planetary sciences (Safronov and Zvjagina, 1969). As is well known, the stable equilibrium state does not exist for celestial objects in circular orbits inside the Roche limit. However, the Roche limit is not practically a good criterion in many cases because the celestial objects possibly take orbits that are eccentric or even parabolic. For parabolic or the hyperbolic orbits with a perigee that is closer than the Roche limit, tidal disruption of the planetesimals have been examined by many authors. Tidal disruption of viscid planetesimals was examined by Mizuno and Boss (1985) using a grid-based hydrodynamics code, and they showed that highly viscous planetesimals would not disrupt. Boss et al. (1991) investigated tidal diruption on inviscid planetesimals using an SPH code. They showed that planetesimals should pass inside the Roche limit to be disrupted. However, according to Boss et al. (1991), the resolution of the target body is not sufficient to describe grazing collisions. In order to grasp the general behavior of the collisional phenomena, we need to simulate and analyze them in a wide range and with fine resolutions.

Collisions and tidal interactions between planetesimals were surveyed by Watanabe and Miyama (1992) to obtain the coalescence rate of planetesimals using the SPH code. These researchers showed that the enlargement factor of the coalescence radius takes 1.7 in uniform density distribution and zero initial relative velocity. The cases of collisions between protoplanets, namely self-gravitating bodies that have density gradients inside of them, have to be examined by new numerical simulations.

This is the first report to be published on our studies to find a theoretical model of mass redistribution in collisions between planetary bodies. The mode should be produced from numerical results among a wide range collisional parameters. The principal aim of this paper is to establish the approach to use for the SPH numerical simulations to pursue collisions between protoplanets and to evaluate quantitatively redistributions of the mass and the angular momentum. Generally speaking, the collisional process is governed by three major parameters: the mass ratio between colliding two bodies, the initial relative velocity, and the impact parameter. In this paper, as a first step, we perform the collisional simulations by changing only the impact parameter.

The assumptions will be presented in Section 2 as well as the method of the numerical simulation and the analysis of the numerical results. In Section 3, the results of the numerical simulations will be shown in detail. Firstly, typical examples will be presented over a wide range of the impact parameter. Secondly, the method of quantitative analysis on the redistribution of mass will be explained. Finally, the redistributions of the mass and the angular momentum of the impactor on the numerical results will be presented. At the same time, the application to the collisional cross section is presented as an example of the application of our numerical results. Conclusions and discussions will be presented in Section 4.

\section{Assumptions and Numerical Procedures \\ 2.1 Assumptions}

In order to pursue impact phenomena between two gravitating bodies, we consider two fluid spheres. We call the larger sphere "target" and the smaller, "impactor". Variables on the target are denoted by " $t$ " and those on impactor by "i". For simplicity, the following assumptions are made in this study:

1) The two spheres are in hydrostatic equilibrium and are not rotating initially.

2) Initially, the relative motion between the two bodies is hyperbolic.

3) The equation of state is given simply by the polytrope,

$$
P=K \rho^{\gamma}
$$


where $P$ and $\rho$ are the pressure and the density, respectively. The polytropic index $\gamma$ is fixed to be 4 in this study. Variations in $\gamma$ will be taken into account after this work.

\subsection{Numerical procedures}

As a numerical method of hydrodynamical calculations, we employ the Smoothed Particle Hydrodynamics (SPH) method (Monaghan, 1992). It is a fully Lagrangian method, like $N$-body simulations. Since being introduced by Lucy (1977) and Gingold and Monaghan (1979), the SPH method has been used in many astrophysical studies.

In the SPH method, fluid is described by a swarm of particles which move under the constants of the equation of motion. For each particle we introduce a kernel $W\left(r_{j}, h_{j}\right)$, which describes the hypothetical density distribution of the fluid around the $j$-th particle. The argument $r_{j}$ represents a distance from the $j$-th particle and $h_{j}$ is a characteristic length of the distribution called "a smoothing length". In this study, we employ a spherically symmetric kernel called the $B$-spline kernel (Monaghan and Lattanzio, 1985), which is defined as follows:

$$
W(r, h)=\frac{1}{4 \pi h^{3}} \begin{cases}3\left(\frac{r}{h}\right)^{3}-6\left(\frac{r}{h}\right)^{2}+4 & \text { for } 0 \leq \frac{r}{h}<1 \\ \left(2-\frac{r}{h}\right)^{3} & \text { for } 1 \leq \frac{r}{h}<2 \\ 0 & \text { for } 2 \leq \frac{r}{h}\end{cases}
$$

Using this kernel, the spatial mass density, $\rho(\boldsymbol{x})$, at a position $\boldsymbol{x}$ is expressed as

$$
\rho(\boldsymbol{x})=\sum_{j=1}^{N} m_{j} W\left(\left|\boldsymbol{x}-\boldsymbol{x}_{j}\right|, h_{j}\right)
$$

where $m_{j}$ and $\boldsymbol{x}_{j}$ are the mass and the position of $j$-th particle, respectively.

In order to describe fluid density contrast stably and reasonably, we adopt the smoothing length which associates with an individual particle, and we should change it as variables. In practice, $h_{j}$ is set so that the particle number within a sphere of radius $h_{j}$ around the $j$-th particle becomes an almost constant value, $N_{\mathrm{SPH}}$, for all SPH particles. However, if we impose this condition strictly, $h_{j}$ oscillates violently in some cases. To suppress this artificial oscillation, we introduce a convergence parameter, $h_{\alpha}$. Thus, we use the smoothing length of the $j$-th particle at time $t=t^{n+1}$, namely $h_{j}^{n+1}$, obeying the following form:

$$
h_{j}^{n+1}=h_{j}^{n}\left(h_{\alpha}+\left(1-h_{\alpha}\right)\left[\frac{N_{\mathrm{SPH}}}{n_{\mathrm{SPH}}}\right]^{1 / 3}\right) \text {, }
$$

where $n_{\mathrm{SPH}}$ is the particle number within a sphere of radius $h_{j}$ around the $j$-th particle at time $t=t^{n}$. In this study, we choose $N_{\mathrm{SPH}}=32$ and $h_{\alpha}=0.2$.

In the standard SPH method, the momentum equation of the $j$-th smoothed particle is given by (see Monaghan,
1992)

$$
\begin{aligned}
m_{j} \frac{\mathrm{d} \boldsymbol{v}_{j}}{\mathrm{~d} t}= & \sum_{k=1}^{N} m_{j} m_{k}\left(\frac{P_{j}}{\rho_{j}^{2}}+\frac{P_{k}}{\rho_{k}^{2}}+\Pi_{j k}\right) \frac{\boldsymbol{r}_{j k}}{r_{j k}} \\
& \cdot \frac{\mathrm{d}}{\mathrm{d} r} W\left(r_{j k}, \frac{h_{j}+h_{k}}{2}\right) \\
& +G \sum_{k=1}^{N} m_{j} M_{k}\left(r_{j k}\right) \frac{\boldsymbol{r}_{j k}}{r_{j k}{ }^{3}},
\end{aligned}
$$

where

$$
\boldsymbol{r}_{j k}=\boldsymbol{x}_{j}-\boldsymbol{x}_{k}
$$

and

$$
r_{j k}=\left|\boldsymbol{r}_{j k}\right|
$$

The origin of coordinates is at the center of mass of the two spheres and $x-y$ plane coincides with their orbital plane. Furthermore, $G, v_{j}, P_{j}$, and $\rho_{j}$ are the gravitational constant, the velocity, the pressure, and the density evaluated at the position of $j$-th particle, respectively. In the first term on the right hand side of Eq. (6), the first and the second terms in the parenthesis represent the pressure gradient force which is caused by neighbor particles (see Appendix A). The third term in the parenthesis, $\Pi_{j k}$, denotes the artificial viscosity to describe shock waves, which is defined as (Monaghan, 1992)

$$
\Pi_{j k}= \begin{cases}\frac{-\alpha c_{j k} \mu_{j k}+\beta \mu_{j k}^{2}}{\rho_{j k}} & \text { for }\left(\boldsymbol{v}_{j}-\boldsymbol{v}_{k}\right) \cdot \boldsymbol{r}_{j k}<0, \\ 0 & \text { for }\left(\boldsymbol{v}_{j}-\boldsymbol{v}_{k}\right) \cdot \boldsymbol{r}_{j k}>0,\end{cases}
$$

where

$$
\mu_{j k}=\frac{h_{j k}\left(\boldsymbol{v}_{j}-\boldsymbol{v}_{k}\right) \cdot \boldsymbol{r}_{j k}}{\boldsymbol{r}_{j k}^{2}+0.01 h_{j k}^{2}} .
$$

According to Monaghan (1992), we put $\alpha=1$ and $\beta=2$ in the present study. Furthermore, $c_{j k}, h_{j k}$, and $\rho_{j k}$ are given as

$$
\begin{aligned}
c_{j k} & =\frac{c_{j}+c_{k}}{2}, \\
h_{j k} & =\frac{h_{j}+h_{k}}{2},
\end{aligned}
$$

and

$$
\rho_{j k}=\frac{\rho_{j}+\rho_{k}}{2}
$$

respectively, where $c_{j}$ is the sound velocity at $\boldsymbol{x}=\boldsymbol{x}_{j}$ defined by

$$
c_{j}=\sqrt{\frac{\gamma P_{j}}{\rho_{j}}} .
$$

The second summation term on the right hand side of Eq. (6) denotes the self-gravity forces acting on the $j$-th particle. In this expression, $M_{k}\left(r_{j k}\right)$ represents a function to correct mutual over-wrapping of neighbor particles. 
The function $M_{k}\left(r_{j k}\right)$ is given by (Gingold and Monaghan, 1977)

$$
M_{k}\left(r_{j k}\right)=m_{k} \begin{cases}\frac{1}{2}\left(\frac{r_{j k}}{h_{k}}\right)^{6}-\frac{6}{5}\left(\frac{r_{j k}}{h_{k}}\right)^{5}+\frac{4}{3}\left(\frac{r_{j k}}{h_{k}}\right)^{3} \\ \left.-\frac{1}{15}+\frac{8}{3}\left(\frac{r_{j k}}{h_{k}}\right)^{3}-3\left(\frac{r_{j k}}{h_{k}}\right)^{4}+\frac{6}{5}\left(\frac{r_{j k}}{h_{k}}\right)^{6}\right)^{5} \\ \text { for } 0 \leq \frac{r_{j k}}{h_{k}}<1, \\ \text { for } 1 \leq \frac{r_{j k}}{h_{k}}<2, \\ \text { for } 2 \leq \frac{r_{j k} .}{h_{k}} .\end{cases}
$$

Note that Eq. (6) is symmetric with respect to $j$ and $k$ and, so, the equation of motion guarantees the linear and the angular momentum conservations.

In addition to the equations presented above, we need an equation of thermodynamical relation between the pressure and the density. As explained in assumption (3), we use a simple polytropic equation of state, i.e., Eq. (2) with $\gamma=4$. The coefficient $K$ in Eq. (2) is determined as follows.

Let us express density and pressure at the center of a polytropic gravitating body with the mass and radius being equal to those of the Earth as $\rho_{0}$ and $P_{0}$, respectively. By imposing a condition that the central density $\rho_{0}$ and the central pressure $P_{0}$ satisfy Eq. (2), we determine the coefficient $K$. Namely, we can express $K$ as

$$
K=b(\gamma) \frac{G M_{\oplus}^{2}}{R_{\oplus}^{4}}\left(a(\gamma) \frac{M_{\oplus}}{\frac{4}{3} \pi R_{\oplus}^{3}}\right)^{-\gamma}
$$

where $a(\gamma)$ and $b(\gamma)$ are numerical constants given for the polytropic index $\gamma$ (see Chandrasekhar, 1967). In the present case where $\gamma=4, a(\gamma)$ and $b(\gamma)$ are equal to 1.506 and 0.174 , respectively. Furthermore, $M_{\oplus}$ and $R_{\oplus}$ are the mass and the radius of the Earth, respectively. In this work, we are interested in collisions between an Earth-mass object and protoplanets in the region of terrestrial planets, namely, the Venus, Earth, and Mars orbit. Therefore, we apply $K$ on the basis of the Earth properties. In this work, the same $K$ and $\gamma$ are applied to the target and the impactor. Of course, the polytropic relation with one pair of the index $\gamma$ and the $K$ can not represent precisely the internal structure of planets, even if we concentrate on only terrestrial bodies. For example, applying this $K$ to the celestial object that has mass equal to that of the Mars, we can not obtain the radius of the body equal to the radius of Mars. Its radius becomes 1.08 times larger than the real radius of Mars. That is why we choose the polytropic relation.

The redistribution of mass and angular momentum brought about by collisions is our main theme. The redistribution should be examined under various conditions and, in this work, the effect from a minimum distance between two bodies is examined. After this study is completed, the effects from other parameters will be examined. Next we focus on the mass ratio between the target and impactor, and we examine the effect caused by the density profile inside the two bodies.

Density profiles inside proto-planetary bodies depend on a number of conditions, with the most important being the difference in its composition. Needless to say, even with the same composition, the degree of central concentration will change in time with evolution or, precisely speaking, with differentiation inside the body. Therefore, if we plan to make a table of redistribution of mass and angular momentum that is applicable to planetary accumulation processes, we have to prepare numerical simulations adapting for each of a number of cases. It is therefore difficult, possibly impossible, to represent all the cases of collisions with a real internal structure model. The reason why we choose the expression of internal density structure by the usage of polytropic relation is mainly to overcome this difficulty.

The polytropic model enables us to change the internal structure easily and systematically. For example, the degree of central concentration can be controlled by changing $\gamma$. Studies based on systematic surveys using polytropic models with changing $\gamma$ and $K$ should be more effective and realistic in terms of obtaining the model of redistributions that is applicable for planetary formation processes than those based on some realistic or detailed equation of states for solid material, for examples Tillotson equations (Tillotson, 1962). So, in this work, as a first step of our studies, we choose an equation of state with one pair of $\gamma$ and $K$.

\subsection{Initial conditions}

As a target, we consider terrestrial size body, of which mass $M_{\mathrm{t}}$ and radius $R_{\mathrm{t}}$ are just equal to $1 M_{\oplus}$ and $1 R_{\oplus}$, re-

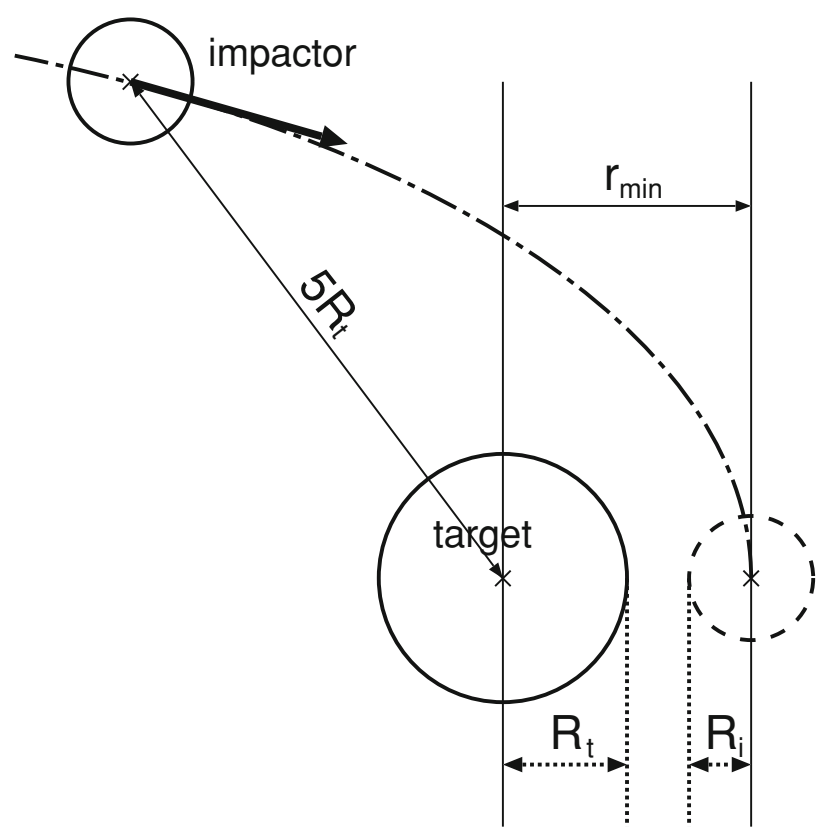

Fig. 1. Schematic picture of the initial condition in a target centered coordinate. The dashed-and-dotted line is a parabolic orbit of the impactor which is assumed as a solid body. The minimum distance $r_{\min }$ is defined as the distance between the target and the impactor at a time of passage of pericenter. 
spectively. The mass of the impactor $M_{\mathrm{i}}$ is also fixed to be $0.06 M_{\oplus}$ in this paper (the corresponding radius $R_{\mathrm{i}}$ is equal to $\left.0.495 R_{\oplus}\right)$. In numerical simulations, the initial distance between the two bodies is taken to be $5 R_{\mathrm{t}}$ to save the computational load and to guarantee the validity of assumption (1) - that the two bodies are initially in a spherically symmetric hydrostatic equilibrium.

To complete the initial kinematic conditions of the two bodies, we impose the following two conditions. First, the minimum distance between the two bodies, $r_{\text {min }}$, is changed in a range from $0.1\left(R_{\mathrm{t}}+R_{\mathrm{i}}\right)$ to $2\left(R_{\mathrm{t}}+R_{\mathrm{i}}\right)$. The parameter $r_{\text {min }}$ is defined as the pericenter distance of a relative Keplerian (parabolic) orbit between two hypothetical points of mass with masses $M_{\mathrm{t}}$ and $M_{\mathrm{i}}$ (see Fig. 1). Secondly, the center of mass of the two bodies is set so as to remain on the origin of coordinates.

In this study, the number of SPH particles is fixed to 21,200, and all the SPH particles have the same mass. As such, the target and the impactor consist of 20,000 and 1,200 particles, respectively, because of mass ratio, $M_{\mathrm{i}} / M_{\mathrm{t}}=0.06$. To check the uncertainties among results with different number of particles, we also calculate simulations using 2,120 particles and 212,000 at $r_{\min } /\left(R_{\mathrm{t}}+R_{\mathrm{i}}\right)=$ 0.5. Comparing results in those three cases, we checked the differences in the redistribution of the mass and the angular momentum due to the difference in number of particles. The results are not same for the simulation using 2,120 particles and 212,000 particles. However, two simulations with 212,000 and 21,200 SPH particles give the same results on the redistributions. Therefore, we conclude that 21,200 particles are enough to calculate the redistribution of the mass and the angular momentum.

\subsection{Aggregate analysis}

As readily conjectured and described later in this report in detail, collisional products change in a complicated manner with the minimum distance, $r_{\min }$. In order to quantitatively pigeonhole collisional products found by numerical simulations, we first divide all SPH particles into three categories namely, particles constituting the central body, particles belonging to an aggregate, and isolated particles. When a particle exists inside a sphere with radius $1.1 R_{\mathrm{t}}$, the center of which is placed at the gravity center of the central body, the particle is regarded as that constituting the central body. Since we cannot recognize a priori the position of the gravity center, we determine iteratively the gravity center of the central body when the number of particles belonging the central body increase.

A particle which does not feel pressure from others is called an "isolated" particle. Particle $j$ is regarded as an isolated particle when the condition,

$$
\begin{aligned}
& \left|\sum_{k} m_{j} m_{k}\left(\frac{P_{j}}{\rho_{j}^{2}}+\frac{P_{k}}{\rho_{k}^{2}}+\Pi_{j k}\right) \frac{\mathrm{d}}{\mathrm{d} r} W\left(r_{j k}, \frac{h_{j}+h_{k}}{2}\right)\right| \\
& <\varepsilon_{\mathrm{f}} \frac{\mathrm{G} M_{\mathrm{c}} m_{j}}{\left|\boldsymbol{r}_{\mathrm{c}}-\boldsymbol{r}_{j}\right|^{2}}
\end{aligned}
$$

is satisfied where $M_{\mathrm{c}}$ and $\boldsymbol{r}_{\mathrm{c}}$ are the mass of the central body and the position of its gravity center, respectively. We introduce $\varepsilon_{\mathrm{f}}$ as a small dimensionless parameter denoting the degree of isolation. In the present study, $\varepsilon_{\mathrm{f}}$ is set at 0.01 .

An aggregate is composed of particles which are classified as neither those of the central body nor as isolated particles. Particles $j$ and $k$ coming close to each other in the phase space are considered to belong to one aggregate, namely, two conditions,

$$
r_{j k} / H_{j k}<\varepsilon_{\mathrm{r}}
$$

and

$$
\left(v_{j k} / V_{j k}\right)^{2}<\varepsilon_{\mathrm{v}},
$$

where $\varepsilon_{\mathrm{r}}$ and $\varepsilon_{\mathrm{v}}$ are non-dimensional parameters. We put $\varepsilon_{\mathrm{r}}=0.24$ and $\varepsilon_{\mathrm{v}}=2.0$ in the present study. In the above conditions, $r_{j k}$ and $v_{j k}$ are the distance and the relative velocity between particles $j$ and $k$, respectively. Furthermore, $H_{j k}$ and $V_{j k}$ are the body Hill radius (Nakazawa and Ida, 1988) and the escape velocity from each other, respectively. They are defined by

$$
H_{j k}=\left(\frac{m_{j}+m_{k}}{3 M_{\mathrm{c}}}\right)^{\frac{1}{3}}\left|\boldsymbol{r}_{\mathrm{c}}-\frac{\boldsymbol{r}_{j}+\boldsymbol{r}_{k}}{2}\right|
$$

and

$$
V_{j k}^{2}=\frac{\mathrm{G} m_{0}}{r_{j k}}
$$

where $m_{0}$ is the mass of one SPH particle. By applying the above two conditions ((18) and (19)) on all pairs except isolated particles and particles belonging to the central body, we can add up all the particles constituting each aggregate.

Using the aggregate analysis mentioned above, we can determine the physical properties of each aggregate, such as the mass, the position of the gravity center, and the instantaneous orbital elements of the Keplerian motion around the central body.

Table 1. Classification of aggregates. In the table, $a$ and $e$ are the semimajor axis and the eccentricity of the instantaneous orbit of an aggregate, respectively. Furthermore, $R_{\mathrm{c}}, R_{\mathrm{a}}$, and $R_{\mathrm{h}}$ are the radii of the central body, the aggregate, and the Hill sphere, respectively, and $\boldsymbol{r}_{\mathrm{a}}$ is the relative position between the aggregate and the central body and $v_{\mathrm{a}}$ is the relative velocity.

\begin{tabular}{c|ccc}
\hline class & $\boldsymbol{r}_{\mathrm{a}} \cdot \boldsymbol{v}_{\mathrm{a}}>0$ & $a(1-e)>R_{\mathrm{c}}+R_{\mathrm{a}}$ & $e>1$ or $a(1+e)>R_{\mathrm{h}}$ \\
\hline escaping & true & - & true \\
& false & true & true \\
\hline bounded & - & true & false \\
\hline merging & true & false & false \\
& false & false & - \\
\hline
\end{tabular}


Finally, we classify aggregates into three categories: "an escaping", "a bounded", and "a merging" aggregate. An "escaping" aggregate possesses an orbit of which the pericenter is larger than $R_{\mathrm{c}}+R_{\mathrm{a}}$ and the apocenter is also larger than $R_{\mathrm{h}}$, where $R_{\mathrm{c}}, R_{\mathrm{a}}$, and $R_{\mathrm{h}}$ are radii of the central body, an aggregate, and the Hill sphere, respectively. Such an aggregate is ejected from the sphere of gravitational influence of the central body. A "bounded" aggregate moves along an elliptic orbit, of which the apocenter is smaller than $R_{\mathrm{h}}$ and the pericenter is larger than $R_{\mathrm{c}}+R_{\mathrm{a}}$. The bounded aggregate is really trapped throughout all eternity without a secondary collision with the central body as long as the aggregate is a rigid body and is not influenced by the strong gravity due to other aggregates. A "merging" aggregate is on an elliptic orbit of which pericenter is smaller than $R_{\mathrm{c}}+R_{\mathrm{a}}$ and collides with the central body in one Keplerian circulation. The classification of aggregates mentioned above is summarized in Table 1 in terms of the instantaneous orbital element $a$ and $e$, namely the semimajor axis and the eccentricity of orbit, respectively.

\section{Numerical Simulations}

\subsection{Typical examples of collisional phenomena}

In order to visualize a variety of collisional phenomena, we first study in detail the time evolution of the collisional processes with various $r_{\min }$. In Fig. 2 we present seven snapshots taken from the numerical simulation for the case of $r_{\text {min }}=0.05\left(R_{\mathrm{t}}+R_{\mathrm{i}}\right)$. Panel 2(a) shows the initial state in which the distance between two bodies is equal to $5 R_{\mathrm{t}}$, as mentioned in Section 2.3. From panel 2(b), which shows a configuration of the two bodies before collision, we can see that the impactor deforms owing to the strong tidal force of the target body. Just before the time in panel 2(c), two bodies collide with each other and merge into one body. Since the impactor lodged completely into the target, we cannot identify particles from the impactor in this panel. After the collision, the merged body is strongly deformed owing to the shock waves propagating in it (see panels 2(d) and 2(e)). During the stage from panels 2(e) to $2(\mathrm{~g})$, the merged body continues to oscillate; the kinetic energy of the oscillatory motion dissipates gradually through the artificial viscosity term in Eq. (6) and, as a result, the oscillation is dumped completely until the time in panel $2(\mathrm{~g})$. At the final stage of our numerical simulation, the merged body settles to a state of hydrostatic equilibrium. It takes a figure of an ellipsoid slightly deformed from a sphere because the body rotates slowly.

In Fig. 3, we present the time sequence of the collision for the case of $r_{\text {min }}=0.6\left(R_{\mathrm{t}}+R_{\mathrm{i}}\right)$. From panel 3(b), we see that the impactor approaches the target being deformed by the tidal force of the target similarly to the case of Fig. 2. Just before the time in panel 3(c), the impactor collides directly with the target and, at a time of panel 3(c) $(t=1.003)$, the center of mass of the impactor passes the pericenter of the initial Keplerian orbit. The impactor collides so deeply that a part of the materials near the collisional point is scooped out from the target with the impactor and stretched out. So, after the collision, almost all of the SPH particles originally comprising the impactor and, additionally, a small part of the target particles are ejected with relatively high velocity.
Thereafter, a small portion of these fall down to the target (see panels 3(d) and 3(e)). The ejected particles then go out of the Roche limit and gather gradually with each other to form a large aggregate due to the self-gravity among the ejected particles (see panel 3(f)). The aggregate, however, cannot survive for a long time because the aggregate moves along an elliptic orbit with a relatively high eccentricity around the target. Consequently, the aggregate collides again with the target and is fragmented into a large number of small aggregates. As a result, the crushed aggregate forms a filamentous long arm (see panel $3(\mathrm{~g})$ ). In panel 3(h) we show the results of the aggregate analysis explained in Section 2.4 on the final stage of the collisional simulation. Namely, the arm is resolved into some aggregates of SPH particles that are shown by colored circles in panel 3(h). At the same time, we also show the instantaneous orbits of the aggregates at time $t=14.295$. The colors of the circles denote the destiny of the aggregate, which is explained in Section 2.4. We can identify seven aggregates, of which two take an elliptical orbit around the central body. These are marked by a dark color (magenta). The remaining aggregates, marked by light color (yellow), take hyperbolic orbit. These are the escaping aggregates. The angular momentum transfer from inside to outside indicate that the aggregates orbiting the outer orbit are the escaping ones.

Figure 4 shows the case where $r_{\min }=0.7\left(R_{\mathrm{t}}+R_{\mathrm{i}}\right)$. Since $r_{\min } /\left(R_{\mathrm{t}}+R_{\mathrm{i}}\right)$ changes only 0.1 from the previous case shown in Fig. 3, panels 4 (a) to $4(\mathrm{f})$ are similar to panels 3(a) to 3(f), but the final product of this collision is quite different from that. The impactor grazes by the surface of the target at a time $t=1.028$ (see panel 4(c)) and is stretched out owing to the tidal force from the target (panels 4(d) and 4(e)). Going outside the Roche lobe, the elongated arm begins to gather again into two aggregates owing to the self gravity. Since the two aggregates are on the elliptical orbits around the target, they approach the target and collide with the target once again (see Fig. 4(h)). As a result, both of them merge to the central body.

In Fig. 5 we present a time sequence of a collisional event for the case of $r_{\text {min }}=1.35\left(R_{\mathrm{t}}+R_{\mathrm{i}}\right)$. Although the impactor does not collide directly with the target (see panel 5(c)), until the stage of panel 5(f), the feature of the collision is similar to that of the previous case of $r_{\min }=0.7\left(R_{\mathrm{t}}+R_{\mathrm{i}}\right)$ where the impactor grazes by the target. This means that the collisional process is essentially governed by the tidal force from the target as long as we are concerned with the cases where the impactor grazes by the target or passes through the vicinity of the target. After the passage of the pericenter of the initial Keplerian orbit (panel 5(c)), the impactor is subjected to large stress due to the tidal force and is finally disrupted into a number of small pieces (panels 5(d) and 5(e)) to form an arc around the central body. In the arc, SPH particles near the central body fall onto the central body, whereas the outer part of the arc continues to go away from the central body. As a result, the impactor is stretched out into a thin filament displayed in panel 5(f). Finally the stretched filament fragments due to its self gravity into one large aggregate and a large number of small ones; the mass of the large fragment is about $0.726 M_{\mathrm{i}}$. Based on the 

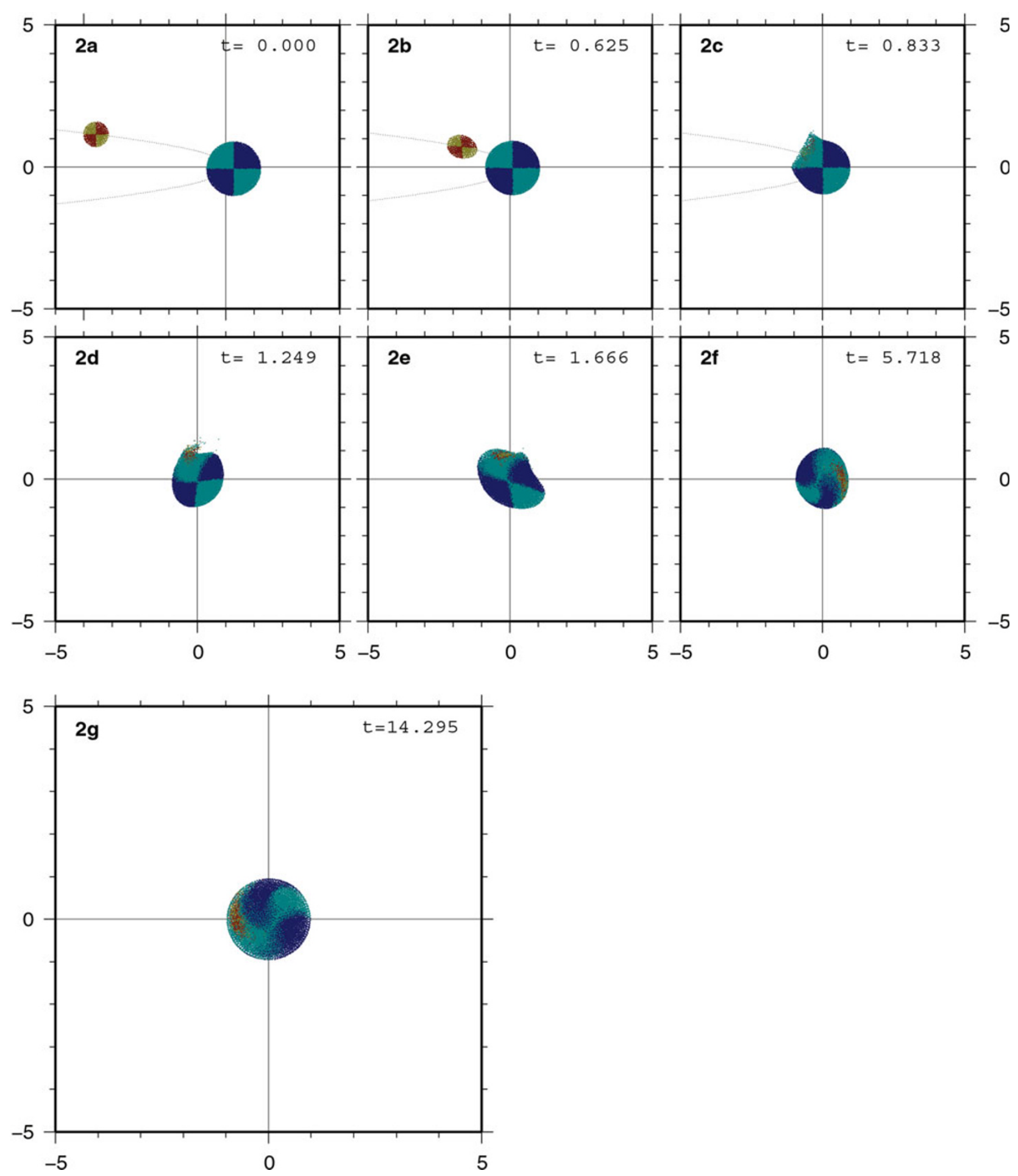

Fig. 2. Time sequence of a collisional event for the case of $M_{\mathrm{i}} / M_{\mathrm{t}}=0.06$ and $r_{\min } /\left(R_{\mathrm{t}}+R_{\mathrm{i}}\right)=0.05$. In each panel, positions of SPH particles are projected to the $x-y$ plane. Particles belonging to the impactor are denoted by dark and light (red and yellow) colors depending on their initial position and, similarly, particles of the target are denoted by dark and light gray (dark blue and light blue color). In each panel the elapsed time, $t$, of the numerical simulation is shown (as for the unit of time, see text).

aggregate analysis mentioned in Section 2.4 , we found that there are one large and six small aggregates in the final stage (panel 5(h)), and that these all escape from the central body along hyperbolic orbits.

In Fig. 6 we show the case of $r_{\min }=1.75\left(R_{\mathrm{t}}+R_{\mathrm{i}}\right)$. In this case, the two bodies do not contact directly, but, owing to strong tidal force from the target, the impactor deforms greatly (panels from 6(c) to 6(e)). Contrary to the previous case (the case of Fig. 5), however, the impactor escapes from the disruption in this case. Only a small number of SPH particles peel off the impactor. Until the deformed impactor obtains a spherical shape, it oscillates dynamically accompanied by a weak dissipation of energy within it. Therefore, the total kinetic energy of the impactor decreases a little. It should be noted that the relative orbit between the two bodies is chosen so that the eccentricity is 

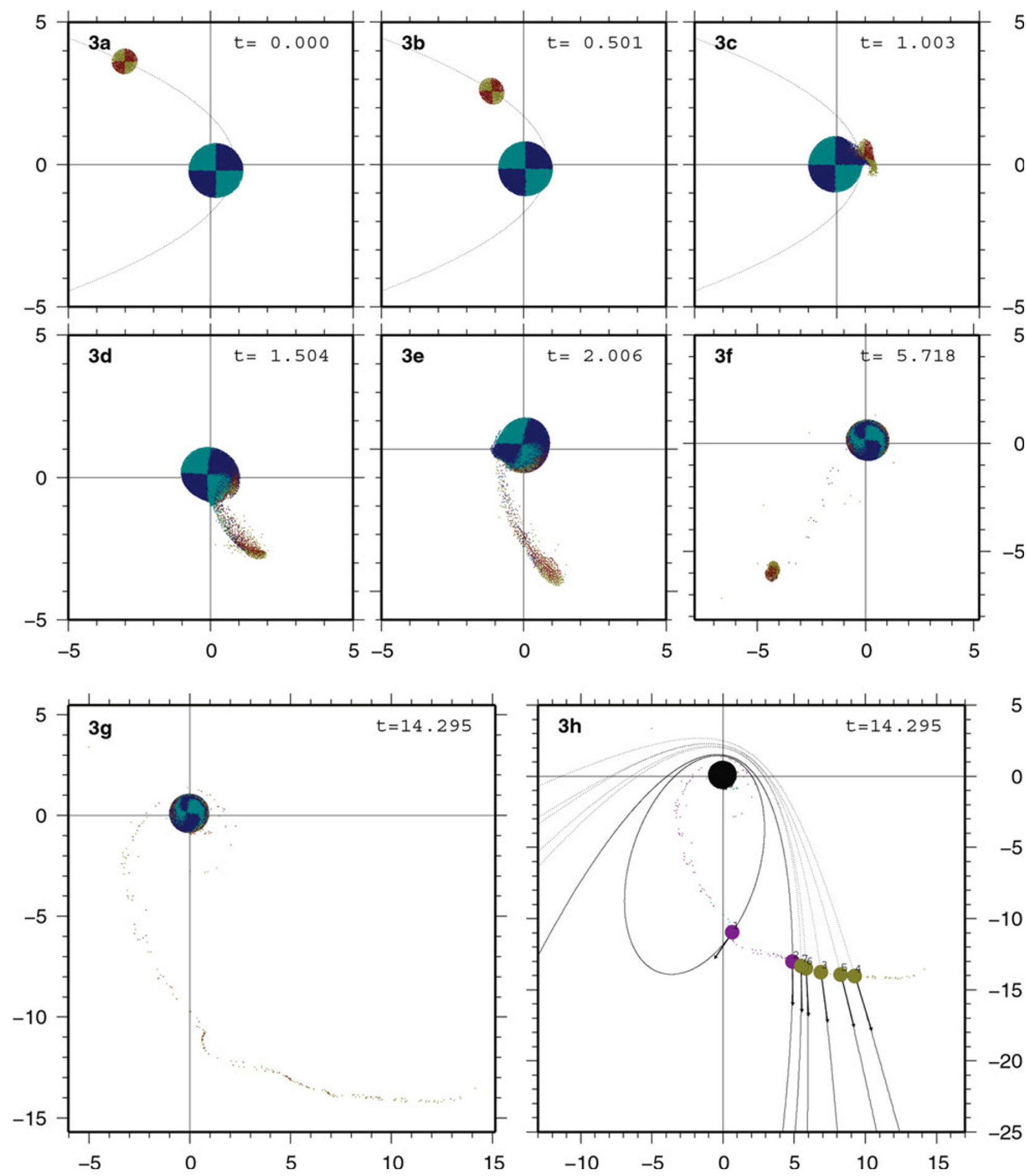

Fig. 3. The same as Fig. 2, but for the case of $r_{\min }=0.6\left(R_{\mathrm{t}}+R_{\mathrm{i}}\right)$. The impactor collides with the target just before the time of panel 3(c). Because of a shallow impact, almost of the impactor materials as well as a small amount of target materials is pulled out (3(d) and 3(e)) and gather each other owing to the self gravity (3(f)). But, the gathered object collides with the target again and a part of it is scattered around the target. Panel $3(\mathrm{~h})$ is the results of the aggregation analysis of the final state. In this case, 8 aggregates (which are denoted by circle) are detected. The color of a circle indicates its destiny; the colors dimgray, darkgray, and lightgray (cyan, magenta, and yellow) denote a merging, a bounded, and an escaping aggregate, respectively. Solid and dotted lines show orbits of aggregates, and arrows denote their velocity.

equal to 1 ; in other words, the initial kinetic energy of the orbital motion is zero. As a result of this very small amount of energy dissipation, the impactor should be trapped within a potential well of the target gravity.

In Fig. 7 we present the case of $r_{\min }=2.5\left(R_{\mathrm{t}}+R_{\mathrm{i}}\right)$. Since the tidal force from the target is small compared with the previous case where $r_{\min }=1.75\left(R_{\mathrm{t}}+R_{\mathrm{i}}\right)$, the deforma- tion of the impactor is weak. In this case, the total kinetic energy also dissipated a little. Through applying the aggregate analysis mentioned earlier, we found that the impactor escapes from the target because its apocenter is far from the Hill radius (Hill, 1878; Nakazawa and Ida, 1988), that is, $240 R_{\mathrm{t}}$. 

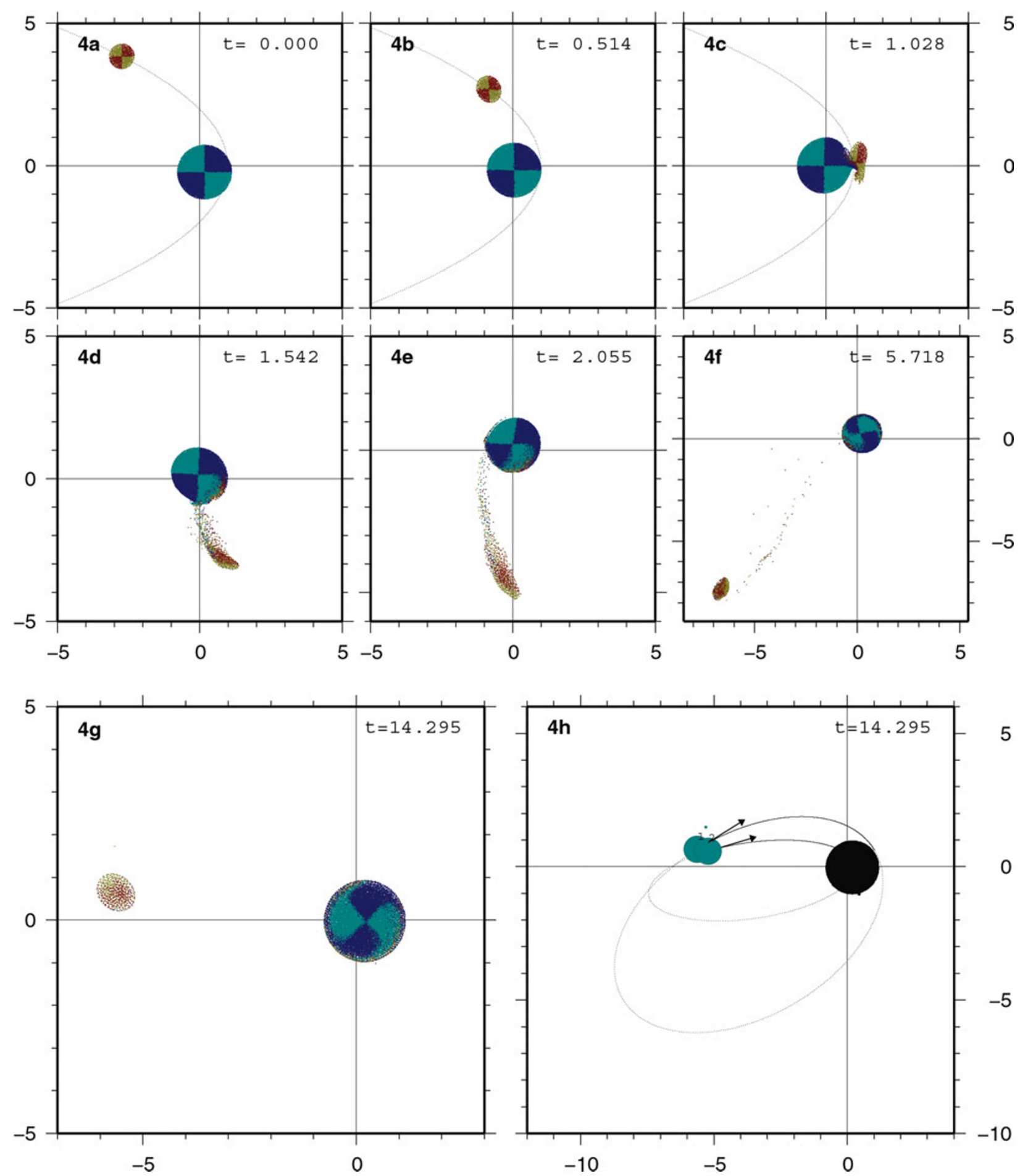

Fig. 4. The same as Fig. 2, but for the case of $r_{\min }=0.7\left(R_{\mathrm{t}}+R_{\mathrm{i}}\right)$. The collisional features are very similar to those of the previous case shown in Fig. 3 until the stage of panel 4(f). But, the final stage of the collision differs from the previous case.

\subsection{Classification of aggregates}

We now consider the classification of aggregates, or in other word, the destiny of aggregates, which are shown in the last snapshots (Fig. 3(g) to Fig. 7(g)), by applying the aggregate analysis mentioned in Section 2.4. The results are summarized in Table 2. We present the number of SPH particles, $n$, that make up the central body and aggregates as well as the numbers of SPH particles coming from the target, $n_{\mathrm{t}}$, and from the impactor, $n_{\mathrm{i}}$. In Table 2, we also show the semimajor axis and the eccentricity of the orbit of each aggregate, from which we judge the destiny of each aggregate shown in the final column.

In the first case mentioned in Section 3, where $r_{\text {min }}=$ $0.05\left(R_{\mathrm{t}}+R_{\mathrm{i}}\right)$, the impactor collides head-on against the target and merges completely as a single body (see Fig. 2). In contrast to this case, we detect seven aggregates around the central body for the case of $r_{\text {min }}=0.6\left(R_{\mathrm{t}}+R_{\mathrm{i}}\right)$ shown in Fig. 3. The sum of the masses of these seven aggregates and the central body is equal to $99.1 \%$ of the total mass. The remaining $0.9 \%$ is distributed into 179 isolated SPH parti- 
Table 2. Physical data of the central body and aggregates for the cases illustrated in Fig. 2(h) to Fig. 7(h). Index shows a label number of an aggregate written in Fig. 2(h) to Fig. 7(h) and $n, n_{\mathrm{t}}$ and $n_{\mathrm{i}}$ are the total number of SPH particles constituting a central body or an aggregate, the number of particles come from the target, and that from the impactor, respectively. The orbital elements $a$ and $e$ are the semimajor axis and the eccentricity of the aggregate, where $a$ is measured by $R_{\mathrm{t}}$. In the last column we present the class of an aggregate judged by the criterion in Table 1 .

\begin{tabular}{|c|c|c|c|c|c|c|c|}
\hline$r_{\min } /\left(R_{\mathrm{t}}+R_{\mathrm{i}}\right)$ & Index & $n$ & $n_{\mathrm{t}}$ & $n_{\mathrm{i}}$ & $a$ & $e$ & Class in Table 1 \\
\hline 0.05 & $\mathrm{C}$ & 21200 & 20000 & 1200 & - & - & - \\
\hline \multirow[t]{8}{*}{0.60} & $\mathrm{C}$ & 20960 & 19996 & 964 & - & - & - \\
\hline & 1 & 30 & 0 & 30 & 8.1 & 0.824 & bounded \\
\hline & 2 & 15 & 0 & 15 & 69.5 & 0.978 & bounded \\
\hline & 3 & 6 & 0 & 6 & 19.9 & 1.108 & escaping \\
\hline & 4 & 4 & 0 & 4 & 8.4 & 1.286 & escaping \\
\hline & 5 & 2 & 0 & 2 & 11.5 & 1.185 & escaping \\
\hline & 6 & 2 & 0 & 2 & 53.2 & 1.038 & escaping \\
\hline & 7 & 2 & 0 & 2 & 204.1 & 1.010 & escaping \\
\hline \multirow[t]{3}{*}{0.70} & $\mathrm{C}$ & 20498 & 19975 & 523 & - & - & - \\
\hline & 1 & 699 & 25 & 674 & 6.3 & 0.783 & merging \\
\hline & 2 & 2 & 0 & 2 & 4.3 & 0.923 & merging \\
\hline \multirow[t]{7}{*}{1.35} & $\mathrm{C}$ & 20258 & 20000 & 258 & - & - & - \\
\hline & 1 & 871 & 0 & 871 & 83.9 & 1.029 & escaping \\
\hline & 2 & 4 & 0 & 4 & 9.3 & 3.328 & escaping \\
\hline & 3 & 3 & 0 & 3 & 7.8 & 2.559 & escaping \\
\hline & 4 & 3 & 0 & 3 & 7.9 & 2.297 & escaping \\
\hline & 5 & 2 & 0 & 2 & 4.9 & 2.623 & escaping \\
\hline & 6 & 2 & 0 & 2 & 174.6 & 1.017 & escaping \\
\hline \multirow[t]{2}{*}{1.75} & $\mathrm{C}$ & 20003 & 20000 & 3 & - & - & - \\
\hline & 1 & 1188 & 0 & 1188 & 48.5 & 0.946 & bounded \\
\hline \multirow[t]{2}{*}{2.50} & $\mathrm{C}$ & 20000 & 20000 & 0 & - & - & - \\
\hline & 1 & 1200 & 0 & 1200 & 1198.2 & 0.997 & escaping \\
\hline
\end{tabular}

cles. As seen from Table 2, the mass of the central body increases slightly (about 5\%) from the initial target mass (which being equal to $1 M_{\oplus}$ ). The largest and the secondary aggregates are composed of 30 and 15 SPH particles, respectively, the other five aggregates are all quite small. The largest aggregate rotates around the central body along an elliptic orbit with $a=8.1 R_{\mathrm{t}}$ and $e=0.824$ and is classified as a bounded aggregate since the pericenter distance is equal to $1.43 R_{\mathrm{t}}$ and the apocenter distance is equal to $14.8 R_{\mathrm{t}}$ and, therefore, smaller than the Hill radius. Though the secondary aggregate is on an elongated elliptic orbit (see Table 2), it can survive as a bounded aggregate because the pericenter and the apocenter distances are equal to $1.51 R_{\mathrm{t}}$ and $137.4 R_{\mathrm{t}}$, respectively. It is important to note here that the pericenters of the two aggregates are within the Roche lobe. Consequently, we expect that the eccentricity of the orbits of these aggregates will decrease gradually owing to the tidal interaction with the central body and, after a while, the aggregates will form a ring around the central body due to the effects of tidal disruption. The other five tiny aggregates escape readily from the Hill sphere because the eccentricities are all larger than 1.

For the case of $r_{\text {min }}=0.7\left(R_{\mathrm{t}}+R_{\mathrm{i}}\right)$ (see Fig. 4), the main collisional products are the central body and an aggregate around the central body. About half of the impactor is transferred into the target and the other half is left as an aggregate on an elliptic orbit. The aggregate labeled as 1 in Table 2 cannot survive as a bounded aggregate because the pericenter distance is equal to $1.37 R_{\mathrm{t}}$ which is smaller than the sum of radii of the central body and the aggregate, $1.44 R_{\mathrm{t}}$. Another tiny aggregate also merges into the central body because of the shortness of the pericenter distance. For the case of $r_{\min }=1.35\left(R_{\mathrm{t}}+R_{\mathrm{i}}\right)$, we find six aggregates; one has a relatively large mass, that is being equal to $75 \%$ of that of the impactor, but the others are very tiny (see Fig. 5). Note that, in this case, the eccentricity of the orbits of all aggregates are larger than 1 and, hence, they escape freely from a sphere of gravitational influence of the central body. In the case of $r_{\min }=1.75\left(R_{\mathrm{t}}+R_{\mathrm{i}}\right)$ (see Fig. 6), after the impactor passes near the target without serious damage, it comes into an orbit for which the eccentricity and semimajor axis are equal to 0.946 and 94.37 , respectively. Since the pericenter distance of the orbit is equal to $2.63 R_{\mathrm{t}}$, the aggregate revolves around the central body stably as a satellite. In the case of $r_{\text {min }}=2.5\left(R_{\mathrm{t}}+R_{\mathrm{i}}\right)$ shown in Fig. 7, the aggregate is judged to escape from the central body because the apocenter is over the Hill sphere of the central body.

\subsection{Redistribution of the mass and the angular mo- mentum}

As seen in the previous subsection, the target hardly loses its appreciable mass in all cases tabulated in Table 2. On the other hand, the mass of the impactor is redistributed in three ways in that it is transferred into the target body, bounded aggregates, and escaping aggregates. In Fig. 8, we show the ratios of the redistribution of the impactor mass into the central body (which is denoted by $M_{\mathrm{m}} / M_{\mathrm{i}}$ ), bounded aggregates (denoted by $M_{\mathrm{b}} / M_{\mathrm{i}}$ ), and escaping aggregates (denoted by $M_{\mathrm{e}} / M_{\mathrm{i}}$ ) as a function of the minimum distance parameter, $r_{\min }$.

Given the behavior of the redistribution of the impactor mass shown in Fig. 8, we can divide the parameter $r_{\min }$ into five ranges, phases $\mathrm{I}$ to $\mathrm{V}$. Phase $\mathrm{I}$ is characterized by the 

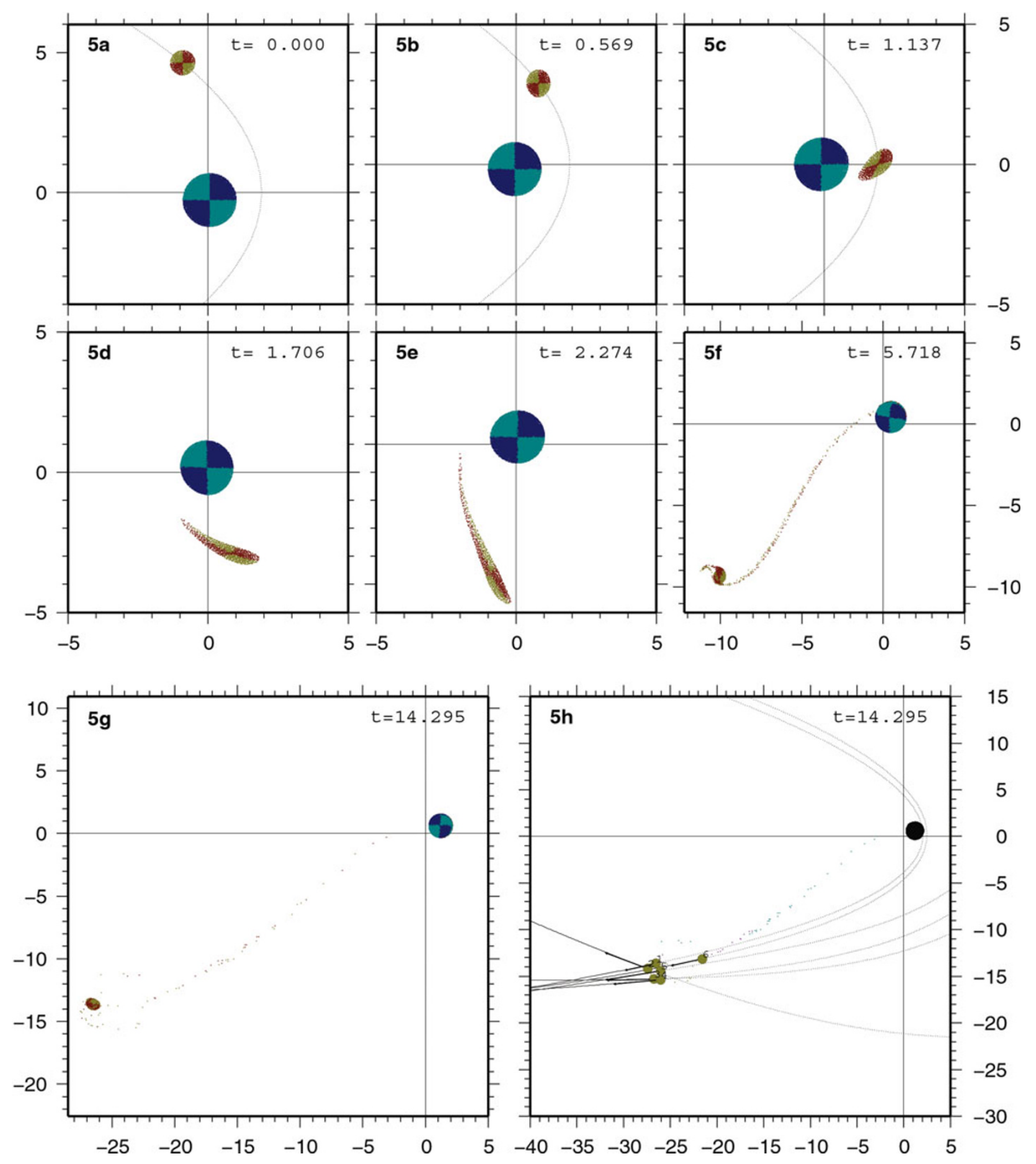

Fig. 5. The same as Fig. 2, but for the case of $r_{\min }=1.35\left(R_{\mathrm{t}}+R_{\mathrm{i}}\right)$. Though the impactor is broken into pieces by the tidal force after the passage of the nearest point (panel 5(c)), almost all of materials constituting originally the impactor gather again (panel 5(g)) and escape from the target.

fact that a large part of the impactor mass is transferred into the target and, hence, the orbital angular momentum of the impactor is transmigrated to the spin angular momentum of the central body. As a result, almost all of the impactor mass is confined within the potential well of the central body. In other words, the mass removed by the escaping aggregates is very small.

In phase II, the behavior of the mass redistribution changes abruptly by a small change in $r_{\min }$. In this phase, a large amount of the impactor remains within the gravitational potential well of the central body as one large ag- gregate. In some cases, the pericenter distance of the orbit of the aggregate is smaller than the sum of the radii of the central body and the aggregate, while in other cases, it is slightly larger. The pericenter distance is very sensitive to a bit change in $r_{\min }$. This is the reason why the mass redistribution changes abruptly against the adopted value of $r_{\min }$. It seems that the boundary of phase I and phase II is located at $r_{\text {min }}=R_{\mathrm{t}}$ in this case. However, it is necessary to simulate a wide range in mass ratio to be able to discuss the general properties of this boundary.

In phase III, the impactor passes nearby the target and it is 

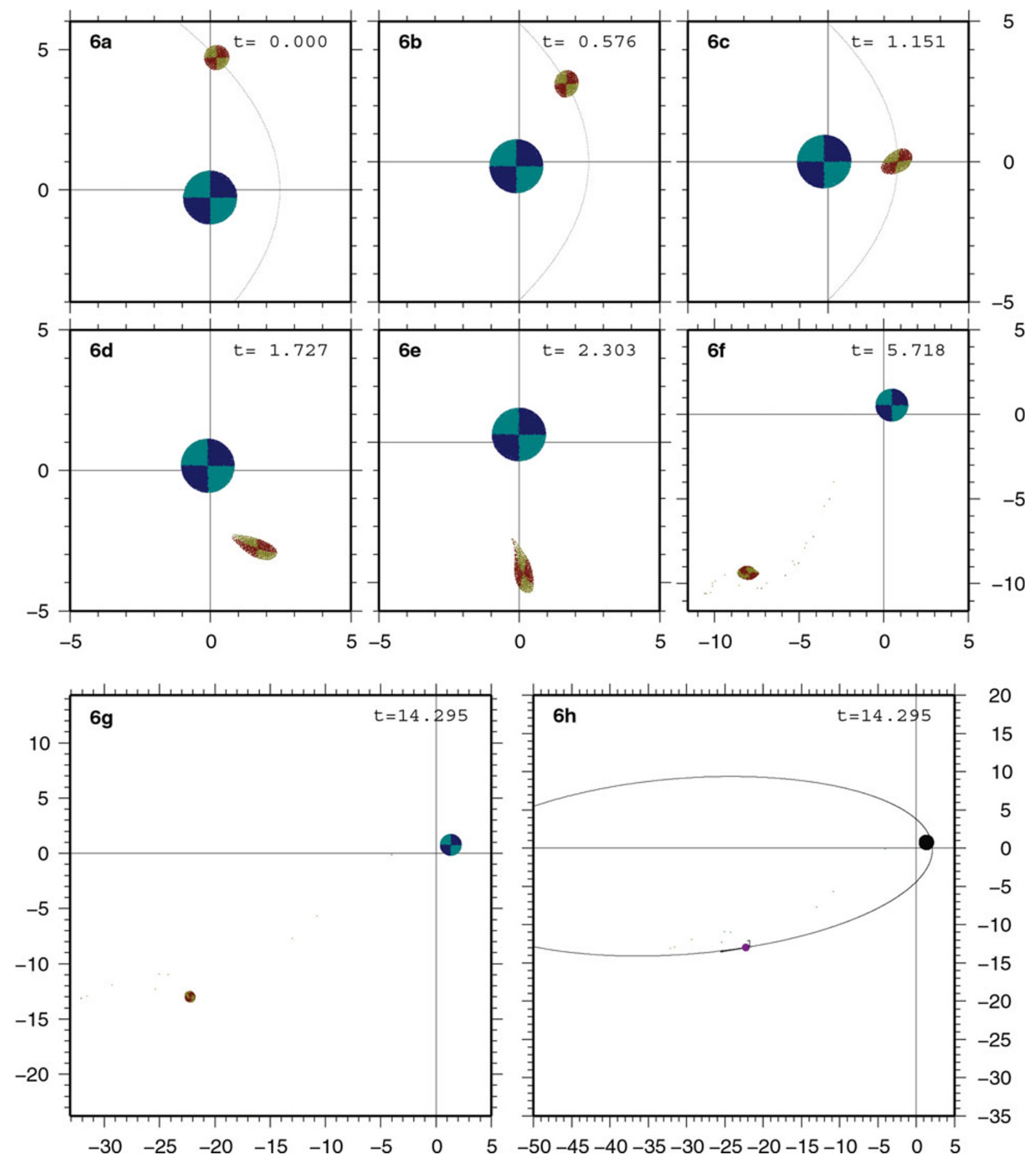

Fig. 6. The same as Fig. 2, but for the case of $r_{\min }=1.75\left(R_{\mathrm{t}}+R_{\mathrm{i}}\right)$. Almost all of materials constituting originally the impactor forms a satellite-like-object trapped within a potential well of the target gravity.

largely deformed by the tidal force of the target. As a result, the impactor is split into several aggregates, some of which escape from the sphere of the gravitational influence of the central body while others merge to the central body. The mass of the impactor is never distributed to the bounded aggregates. In contrast to phase III, phase IV is characterized by a large fraction of bounded aggregates. In this phase, after the impactor is deformed near the target, it oscillates violently and loses energy due to viscous dissipation. As a result, the impactor is captured with the target. The viscous dissipation in phase III is more effective than that in phase
IV. However, in phase III, the largely deformed impactor generates the non-axisymmetric gravitational field that is strong enough to transfer the angular momentum from the inner part to the outer one. This is the reason why a large amount of the mass of the impactor is ejected in phase III.

In phase $\mathrm{V}$, the impactor passes through the target and is ejected as is.

The boundary of phase III, phase IV, and phase V are recognized very sharply on the panel of the bounded mass fraction or escaping mass fraction because these boundaries are determined by the distance of apocenter of the largest 

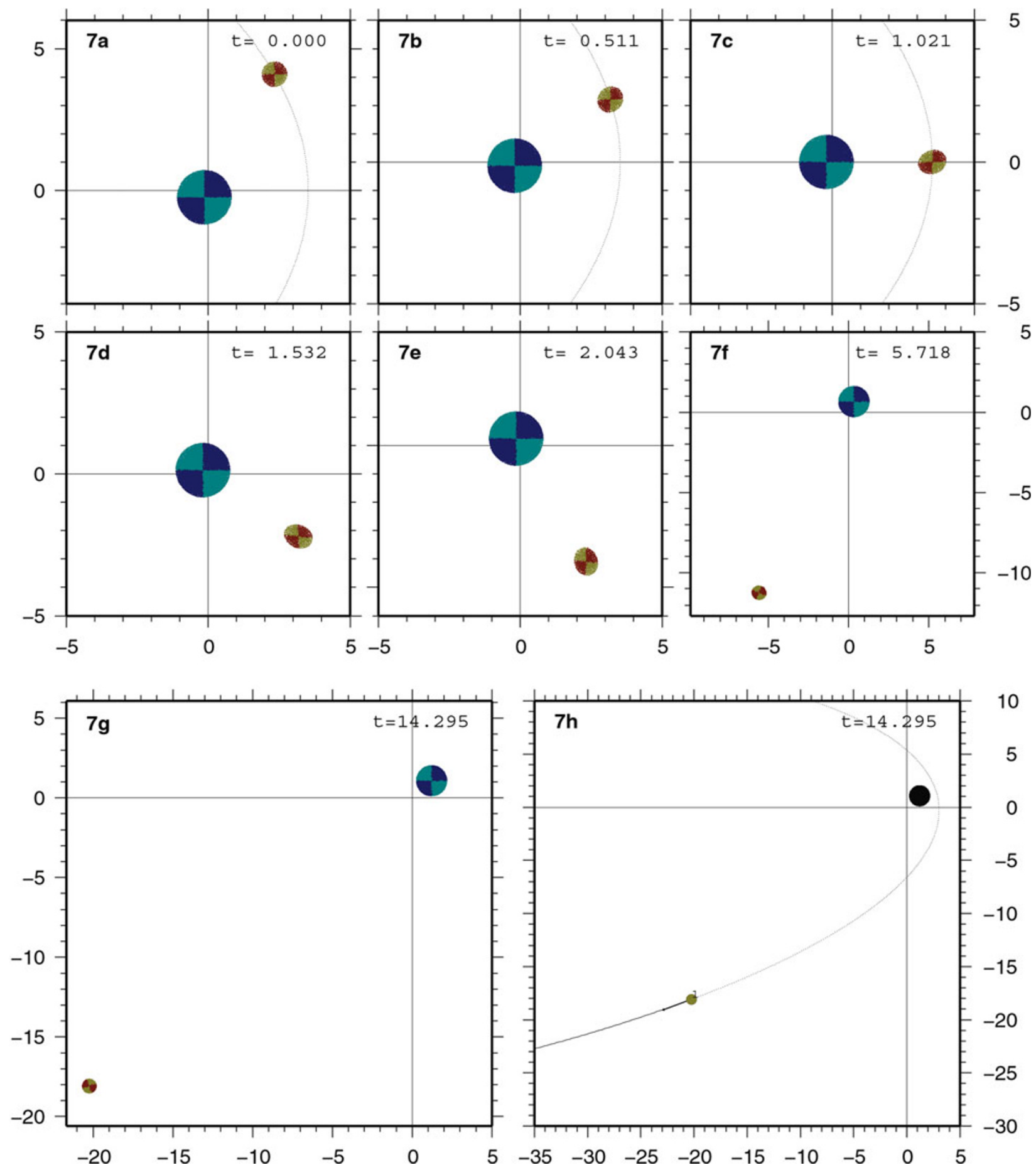

Fig. 7. The same as Fig. 2, but for the case of $r_{\min }=2.5\left(R_{\mathrm{t}}+R_{\mathrm{i}}\right)$. The impactor passes near the target in panel 7(c) and is deformed owing to the tidal force (see panels 7 (d) to 7(f)). After that the impactor recovers its sphericity and escapes from the potential well of the target.

aggregate.

We now see the redistribution of the orbital angular momentum of the impactor. Since both the target and the impactor do not rotate initially, the dynamical system has the angular momentum, $L_{\text {ini }}$, caused by the relative orbital motion, which is given by

$$
L_{\mathrm{ini}}=\frac{M_{\mathrm{i}} M_{\mathrm{t}}}{M_{\mathrm{i}}+M_{\mathrm{t}}} \sqrt{2 \mathrm{G}\left(M_{\mathrm{i}}+M_{\mathrm{t}}\right) r_{\min }},
$$

because, in the present study, the initial relative orbits are all assumed to be parabolic. A part of the orbital angu- lar momentum is transferred to the central body through a direct attachment as well as the gravitational interaction between the target and the impactor. Therefore, the behavior of the redistribution of the angular momentum depends on the collisional phase, i.e., the minimum distance, $r_{\text {min }}$. In Fig. 9, we show the spin angular momentum transmigrated to the central body as a function of $r_{\min }$, as well as the orbital angular momentum of the bounded aggregates and that brought off by the escaping aggregates. Roughly speaking, we can see from Fig. 9 that the angular momentum transferred to the central body decreases with an increase in $r_{\text {min }}$ 


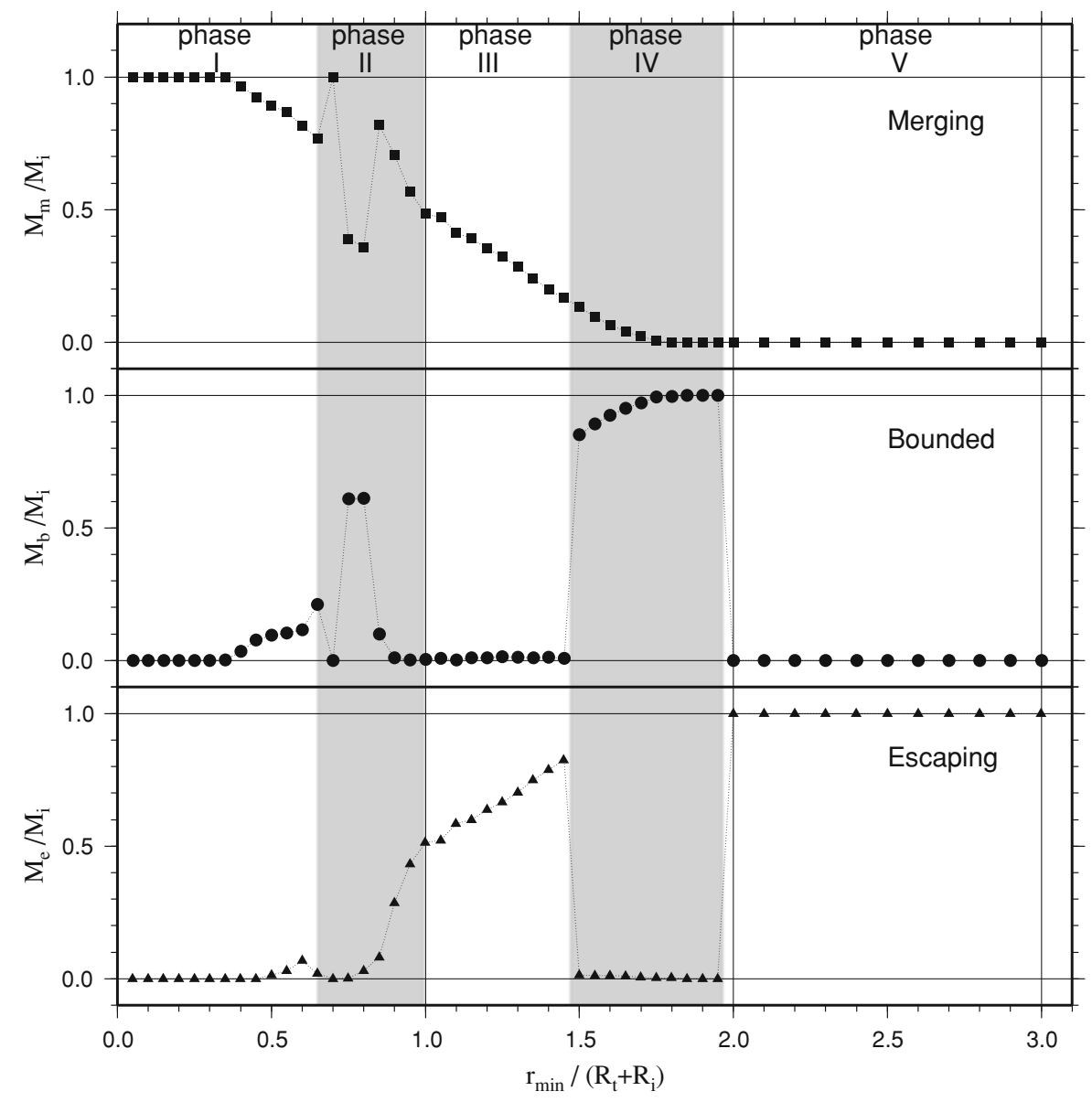

Fig. 8. The ratios of masses merging to the central body, $M_{\mathrm{m}}$, belonging to the bound aggregates, $M_{\mathrm{b}}$, and being taken off by the escaping aggregates, $M_{\mathrm{e}}$ to the impact mass as a function of $r_{\min }$, which is normalized by the sum of radii of the target and the impactor. From the behavior of the redistribution of the impactor mass, we divide the range of $r_{\min }$ into five phases, phase I to $\mathrm{V}$.

and that, as a matter of course, $L_{\mathrm{m}} / L_{\text {ini }}$ behaves very similarly to $M_{\mathrm{m}} / M_{\mathrm{i}}$.

In phase I (where $r_{\min }$ is smaller than $0.65\left(R_{\mathrm{t}}+R_{\mathrm{i}}\right)$ ), the central body receives more than $60 \%$ of the orbital angular momentum, $L_{\text {ini }}$. In phase II, $L_{\mathrm{m}} / L_{\text {ini }}$ (as well as $L_{\mathrm{b}} / L_{\text {ini }}$ ) behaves in a complicated manner against the choice of $r_{\text {min }}=M_{\mathrm{m}} / M_{\mathrm{i}}$. In phase III (i.e., $1.0 \leq r_{\min } /\left(R_{\mathrm{t}}+R_{\mathrm{i}}\right) \leq$ $1.5)$, less than $20 \%$ of the initial orbital angular momentum is added to the central body and the rest is carried away from the system by the escaping aggregates. On the other hand, in phase IV (or V) almost all of the angular momentum is transferred to the bounded aggregates (or the escaping aggregates).

Figure 10 shows the physical properties of the largest aggregate at the final state of the simulations. Panel 10(a) shows the mass of the largest aggregate. We can see that the mass of the largest aggregate is larger than half the mass of the impactor at the initial state for the case of $r_{\min }>R_{\mathrm{t}}+R_{\mathrm{i}}$. In this case, because the impactor does not contact directly with the target, the impactor is disrupted by the tidal force, and the rest of the impactor becomes the largest aggregate at the final state. Panel 10(b) describes the orbital angular momentum of the largest aggregate compared with its initial value. In this study, the orbital angular momentum of the largest aggregate is smaller than that of the impactor at the initial state because the spin of the target and that of the impactor is set to 0 at the initial state. We can see that even in the case when the impactor does not lose its mass (i.e., in phase V), the largest aggregate loses its angular momentum. However, because the angular momentum is transferred by self gravity by means of an arm-like structure from inside to the outside of the arm, the angular momentum does not decrease as much in comparison to the decrease in the mass in phase III. Panel 10(c) describes the specific orbital angular momentum of the largest aggregate compared with its initial value. The specific angular momentum increases largely in phase III compared with phase IV owing to this angular momentum transfer.

To summarize on the angular momentum, there are three effects on the angular momentum to change - the mass loss and the conversion from the orbital angular momentum to spin, and the angular momentum transfer by self gravity to increase angular momentum. The behavior of the redistribution is determined by the balance of these effects.

\subsection{Application to the collisional cross section}

We will now apply our results to the evaluation of the cross section, $\sigma$, which governs the growth of the target (protoplanetary) mass, which is formally written as

$$
\sigma=\int_{0}^{\infty} 2 \pi b \mathrm{~d} b \frac{M\left(r_{\min }\right)}{M_{\mathrm{i}}}
$$




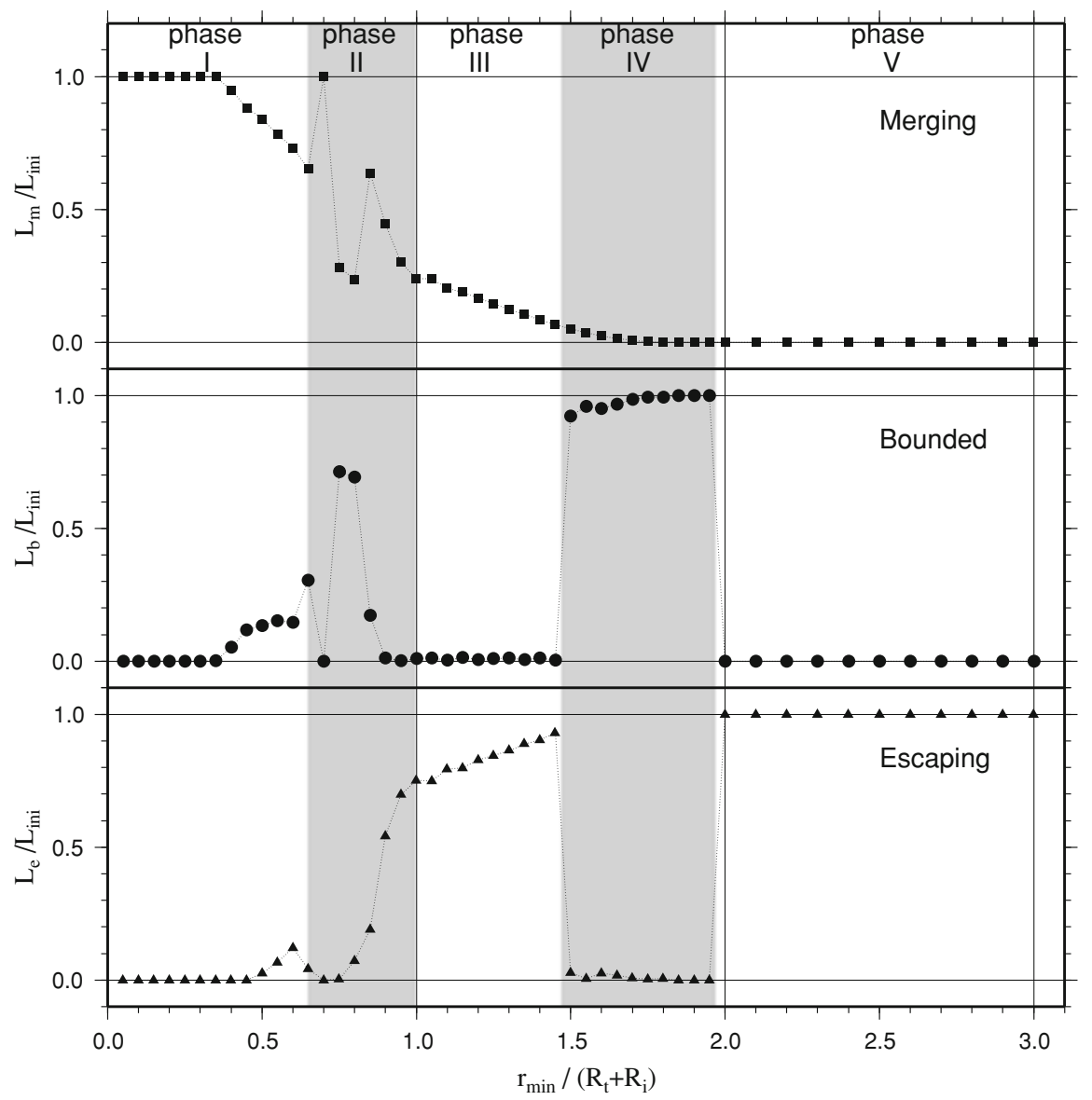

Fig. 9. The ratios of angular momentums merging to the central body, $L_{\mathrm{m}}$, belonging to the bound aggregates, $L_{\mathrm{b}}$, and being taken off by the escaping aggregates, $L_{\mathrm{e}}$ to the total angular momentum as a function of $r_{\min }$, which is normalized by the sum of radii of the target and the impactor.

where $b$ is the so-called impact parameter, $M_{\mathrm{i}}$ is the impactor mass, and $M\left(r_{\min }\right)$ is the coalescent mass to the target by a collision with the minimum distance $r_{\min }$. From the conservation laws of the energy and the angular momentum, we can readily find

$$
b \mathrm{~d} b=\left\{r_{\text {min }}+\frac{v_{\text {esc }}^{2}}{2 v_{\infty}^{2}}\left(R_{\mathrm{t}}+R_{\mathrm{i}}\right)\right\} \mathrm{d} r_{\text {min }},
$$

where $v_{\infty}$ is the relative velocity at a far distance and $v_{\text {esc }}$ is the escape velocity given by

$$
v_{\mathrm{esc}}^{2}=\frac{2 G\left(M_{\mathrm{i}}+M_{\mathrm{t}}\right)}{R_{\mathrm{i}}+R_{\mathrm{t}}}
$$

Thus, the cross section $\sigma$ is rewritten as

$$
\sigma=\pi\left(R_{\mathrm{i}}+R_{\mathrm{t}}\right)^{2}\left\{f_{1}+\left(\frac{v_{\mathrm{esc}}}{v_{\infty}}\right)^{2} f_{2}\right\},
$$

where the coefficients $f_{1}$ and $f_{2}$ are given, respectively, by

$$
f_{1}=\frac{2}{M_{\mathrm{i}}\left(R_{\mathrm{t}}+R_{\mathrm{i}}\right)^{2}} \int_{0}^{\infty} M\left(r_{\text {min }}\right) r_{\text {min }} \mathrm{d} r_{\text {min }}
$$

and

$$
f_{2}=\frac{1}{M_{\mathrm{i}}\left(R_{\mathrm{t}}+R_{\mathrm{i}}\right)} \int_{0}^{\infty} M\left(r_{\min }\right) \mathrm{d} r_{\min } .
$$

Table 3. Cross section of the mass. The coefficients $f_{1}$ and $f_{2}$ of the cross section formula (Eq. (26)) for the two cases where $M$ is equal to $M_{\mathrm{m}}$ and $M_{\mathrm{m}}+M_{\mathrm{b}}$.

\begin{tabular}{lcc}
\hline$M\left(r_{\min }\right)$ & $f_{1}$ & $f_{2}$ \\
\hline$M_{\mathrm{m}}$ & 1.17 & 0.96 \\
$M_{\mathrm{m}}+M_{\mathrm{b}}$ & 2.98 & 1.54 \\
\hline
\end{tabular}

If $M\left(r_{\min }\right)$ is equal to $M_{\mathrm{i}}$ for the case of $r_{\min } \leq R_{\mathrm{t}}+R_{\mathrm{i}}$ and 0 for another case, both $f_{1}$ and $f_{2}$ are reduced to 1 and the cross section of the mass accretion coincides with the Safronov formula (Safronov and Zvjagina, 1969).

As for the coalescent mass to the target, $M\left(r_{\text {min }}\right)$, it seems natural to include the merging mass $M_{\mathrm{m}}$ found in our present simulations (see Fig. 8). However, there is another way to include $M\left(r_{\min }\right)$, namely, there is a possibility that bounded aggregates contribute to the increment of the target mass for the case where the growth of the protoplanets proceeds in a gaseous environment (i.e., in a gas of the solar nebula). In such a case, we should take $M_{\mathrm{m}}+M_{\mathrm{b}}$ for $M\left(r_{\min }\right)$. In Table 3 , the coefficients $f_{1}$ and $f_{2}$ are shown for the two cases mentioned above. When we consider only $M_{\mathrm{m}}$ for the coalescent mass, $M\left(r_{\min }\right)$, both $f_{1}$ and $f_{2}$ are almost equal to 1 and, therefore, the cross section can be expressed simply by the Safronov formula. For the case of $M\left(r_{\min }\right)=M_{\mathrm{m}}+M_{\mathrm{b}}$, on the other hand, $f_{1}$ and $f_{2}$ become large compared to those of the previous case, $f_{1}=3.0$ and 
The mass and the angular momentum of the largest aggregate

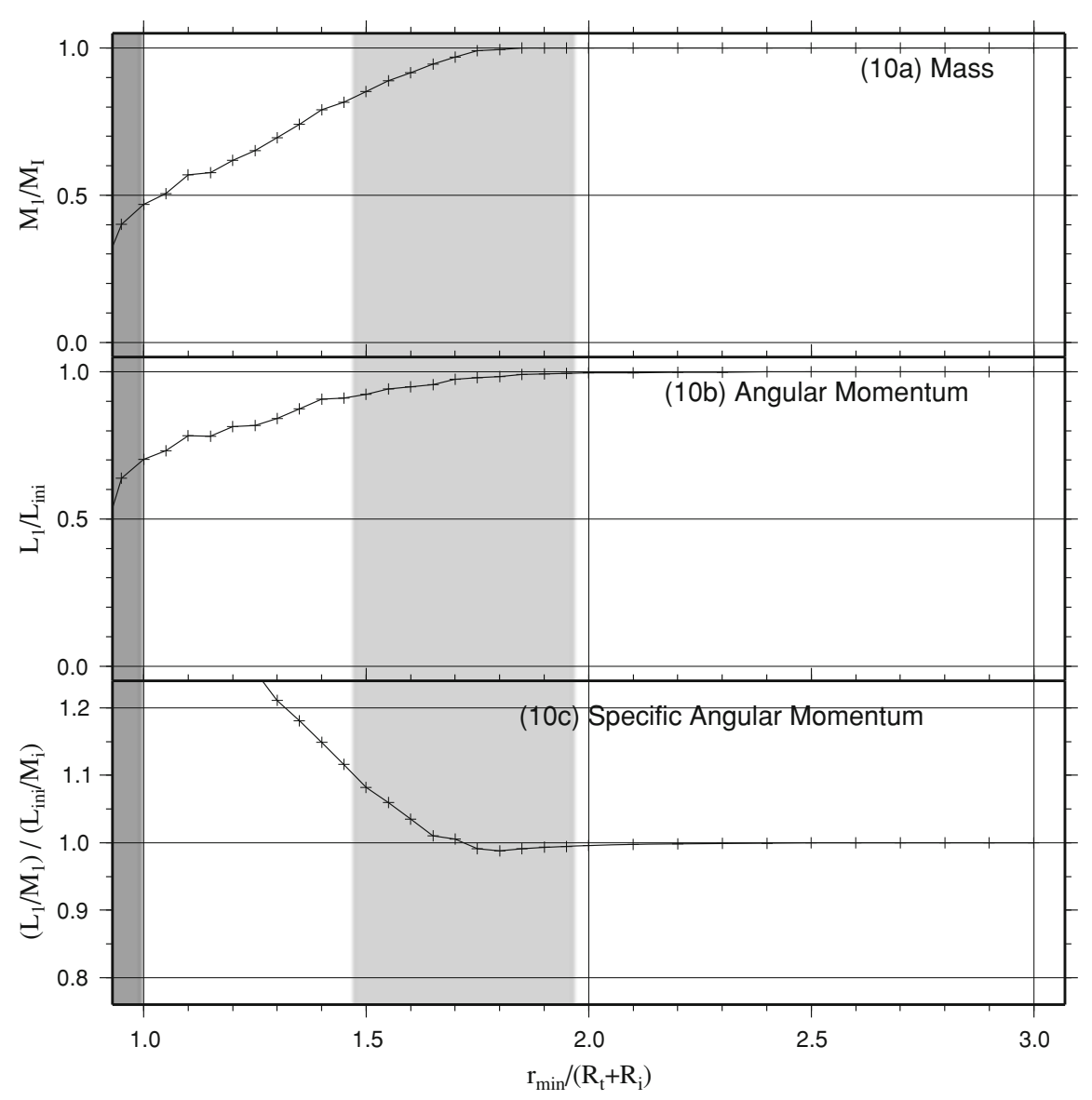

Fig. 10. The mass and the angular momentum of the largest aggregate as a function of $r_{\min }$, which is normalized by the sum of radii of the target and the impactor. (a) the mass of the largest aggregate normalized by the mass of the impactor, (b) the orbital angular momentum of the largest aggregate normalized by that of the impactor at the initial state, and (c) the specific angular momentum of the largest aggregate normalized by that of the impactor at the initial state. In each panel, the shaded zones denote phases which are shown in Fig. 8.

$f_{2}=1.5$. It is interesting to note that, in the second case, we can rewrite Eq. (26) approximately to the following form:

$$
\sigma=\pi\left(R_{\mathrm{i}}+R_{\mathrm{t}}\right)^{2} f^{2}\left(1+\frac{v_{\mathrm{esc}}^{2}}{f v_{\infty}^{2}}\right),
$$

where $f=f_{1}^{1 / 2}$. This means that the effective radius becomes large by a factor of $f(=1.7)$ and that the growth of the protoplanet can be accelerated by a factor of 3 when the planetary growth proceeds in the nebula gas. The value $f=1.7$ is consistent with coalescence radius $f=1.7$ of Watanabe and Miyama (1992) in the case of $\gamma=3$ and $M_{\mathrm{i}} / M_{\mathrm{t}}=0.25$.

Although we carried out the simulations reported here under the condition that $v_{\infty}=0$ we can evaluate the enhancement factors $f_{1}$ and $f_{2}$ because we calculate them using Eqs. (27) and (28) which do not include $v_{\infty}$. In this case, these factors are interpreted to be limiting ones to lowvelocity impacts. These values of $f_{1}$ and $f_{2}$ may be maximum values. We consider that the deformation of gravitating bodies becomes larger as the relative velocity between them becomes smaller. The behavior of impacts under different relative energy conditions is one of the aims of our objects for future work.

\section{Conclusions and Concluding Remarks}

In this study, we have performed impact simulations between hydrostatic equilibrium spheres changing the minimum distance, $r_{\min }$, for the limited cases where the masses of the two spheres are fixed to be $1 M_{\oplus}$ (target) and $0.06 M_{\oplus}$ (impactor) and where the kinetic energy of the two-body system is zero when one body is far from another (i.e., the relative motion is described by a parabolic orbit). Using a number of numerical simulations, we arrive at the following conclusions, which are of great interest.

1) The behavior of the collision (i.e., the products after a collision) varies with $r_{\min }$ continuously as long as we limit ourselves to a limited region of $r_{\min }$, but it changes abruptly at some points of $r_{\text {min }}$.

2) As a result, we can divide the region of $r_{\min }$ into five phases, from phases I to V, as mentioned in Section 3.3. The redistribution of the impactor mass changes continuously in each phase except for phase II, but changes abruptly at boundaries between adjacent phases.

3) The redistribution of the angular momentum resembles the redistribution of the mass. However, accumulation of the angular momentum to the central body is 
less effective than that of the mass because the angular momentum is transferred through the gravitational interaction to the bounded and escaping aggregates.

4) The coalescent cross section, which governs planetary growth, increases by a factor 3 when the masses of the bounded aggregates also contribute to the growth of the protoplanet when the planetary growth goes on within the nebular gas.

We have obtained interesting results on the behavior of collision between two protoplanets. However, our numerical simulations are made under a number of limiting conditions. First, the mass of the target and the impactor are fixed to be $1 M_{\oplus}$ and $0.06 M_{\oplus}$, respectively. Furthermore, we consider only the case where the relative orbit is parabolic initially (i.e., the orbital eccentricity is just equal to 1 ). If we consider a heavier impactor than the one in this study, the target would be largely deformed by the gravitational force of the impactor and friction between the target and the impactor would become effective. Therefore, the impact phase III may disappear.

The behavior of the collisions changes with changing orbital eccentricity $e$. When $e<1$, the relative velocity at the pericenter becomes smaller and the impactor stays during a relatively long time within the Roche lobe. As a result, the impactor should be apt to fragment to form a massive disk around the target. In the case where $e \gg 1$, i.e., the kinetic energy of the relative motion is comparable to or greater than the self-gravitational energy of the target, and the collision product must be quite different from those shown in the present study. The target would suffer serious damage and, as a result, might lose an appreciable amount of mass.

It is also important to perform further simulations with other values of the polytropic index $\gamma$ (see Eq. (2)). When we choose smaller $\gamma$, the target as well as the impactor become soft and, therefore, the impactor should be apt to stretch at the pericenter. However, according to our preliminary results of changing $\gamma$, it would seem that the dependency on the $\gamma$ is quite small.

Our aim is, we have to pursue the collisional phenomena by changing widely the mass ratio $M_{\mathrm{i}} / M_{\mathrm{t}}$, the orbital eccentricity $e$, and the polytropic index $\gamma$. A part of these results will be presented in the next paper.

Acknowledgments. The authors are grateful to Dr. Sei-ichiro Watanabe for his careful review of this article and useful suggestions, and to Dr. Sin-iti Sirono for helpful comments and continuous encouragement. The numerical simulations in this paper were performed with the special purpose hardware, GRAPE-4, and Dr. Ida and Dr. Makino provided assistance when GRAPE-4 was used. This work is partly supported by Grants-in-Aid for Scientific Research, Scientific research (C), 16540382.

\section{Appendix A. Expression of the Pressure Gradient Term in the SPH Framework}

Note that the density at an arbitrary position $\boldsymbol{x}$ is given by (see Eq. (4))

$$
\rho(\boldsymbol{x})=\sum_{j=1}^{N} m_{k} W\left(\left|\boldsymbol{x}-\boldsymbol{x}_{k}\right|, h_{k}\right)
$$

where $m_{k}$ is the mass of $k$-th particle and $W$ is the kernel function given by Eq. (3). Similarly, a physical value $A$ at an arbitrary position $\boldsymbol{x}$ is interpolated as

$$
A(\boldsymbol{x})=\sum_{j=1}^{N} \frac{A_{k} m_{k} W\left(\left|\boldsymbol{x}-\boldsymbol{x}_{k}\right|, h_{k}\right)}{\rho_{k}},
$$

where $A_{k}$ is the value of $A$ at the position of $k$-th particle.

Now, we readily write down the pressure gradient term, $\nabla P / \rho$, evaluated at $j$-th particle as

$$
\left(\frac{\nabla P}{\rho}\right)_{j}=\frac{P_{j}}{\rho_{j}^{2}} \nabla_{j} \rho_{j}+\nabla_{j}\left(\frac{P}{\rho}\right)_{j}
$$

where $\nabla_{j}$ is a differential operator which acts on $\boldsymbol{x}_{j}$. With the help of Eq. (A.2), we have for $(P / \rho)_{j}$

$$
\left(\frac{P}{\rho}\right)_{j}=\sum_{k=1}^{N} \frac{\left(P_{k} / \rho_{k}\right) m_{k} W\left(\left|\boldsymbol{x}_{j}-\boldsymbol{x}_{k}\right|, h_{k}\right)}{\rho_{k}} .
$$

Substituting Eqs. (A.1) and (A.4) into Eq. (A.3), $(\nabla P / \rho)_{j}$ is found to be

$$
\left(\frac{\nabla P}{\rho}\right)_{j}=\sum_{k=1}^{N} m_{k}\left(\frac{P_{j}}{\rho_{j}^{2}}+\frac{P_{k}}{\rho_{k}^{2}}\right) \nabla_{j} W\left(\left|\boldsymbol{x}_{j}-\boldsymbol{x}_{k}\right|, h_{k}\right) .
$$

In order to guarantee the conservation laws of the momentum and the angular momentum, $h_{k}$ in Eq. (A.5) should be replaced by $h_{j k}$ where

$$
h_{j k}=\frac{h_{j}+h_{k}}{2} \text {. }
$$

\section{References}

Agnor, C. and E. Asphaug, Accretion efficiency during planetary collisions, Astrophys. J. Lett., 613, L157-L160, 2004.

Benz, W., W. L. Slattery, and A. G. W. Cameron, The origin of the moon and the single-impact hypothesis. I, Icarus, 66, 515-535, 1986.

Benz, W., W. L. Slattery, and A. G. W. Cameron, The origin of the moon and the single-impact hypothesis. II, Icarus, 71, 30-45, 1987.

Benz, W., A. G. W. Cameron, and H. J. Melosh, The origin of the moon and the single impact hypothesis. III, Icarus, 81, 113-131, 1989.

Boss, A. P., A. G. W. Cameron, and W. Benz, Tidal disruption of inviscid planetesimals, Icarus, 92, 165-178, 1991.

Cameron, A. G. W., The origin of the Moon and the single impact hypothesis V, Icarus, 126, 126-137, 1997.

Cameron, A. G. W., Higher-resolution simulations of the giant impact, in Origin of the earth and moon, edited by R. M. Canup and K. Righter, 555 pp., University of Arizona Press, Tuscon, 2000.

Cameron, A. G. W. and W. Benz, The origin of the moon and the single impact hypothesis. IV, Icarus, 92, 204-216, 1991.

Cameron, A. G. W. and W. R. Ward, The origin of the Moon, in Lunar and Planetary Institute Conference Abstracts, 120-122, 1976.

Canup, R. M., Dynamics of lunar formation, Ann. Rev. Astron. Astrophys., 42, 441-475, 2004a.

Canup, R. M., Simulations of a late lunar-forming impact, Icarus, 168, 433-456, 2004b.

Canup, R. M. and E. Asphaug, Origin of the Moon in a giant impact near the end of the Earth's formation, Nature, 412, 708-712, 2001.

Canup, R. M., W. R. Ward, and A. G. W. Cameron, A scaling relationship for satellite-forming impacts, Icarus, 150, 288-296, 2001.

Chandrasekhar, S., An introduction to the study of stellar structure, 509 pp., Dover, New York, 1967.

Gingold, R. A. and J. J. Monaghan, Smoothed particle hydrodynamicsTheory and application to non-spherical stars, Mon. Not. Roy. Astron. Soc., 181, 375-389, 1977. 
Gingold, R. A. and J. J. Monaghan, Binary fission in damped rotating polytropes. II, Mon. Not. Roy. Atron. Soc., 188, 39-44, 1979.

Hartmann, W. K. and D. R. Davis, Satellite-sized planetesimals and lunar origin, Icarus, 24, 504-514, 1975.

Hayashi, C., K. Nakazawa, and Y. Nakagawa, Formation of the solar system, in Protostars and Planets II, edited by D. C. Black and M. S. Matthews, 1313 pp., University of Arizona Press, Tucson, 1985.

Hill, G. W., Researches in the lunar theory, Am.J. Math., 1, 5-26, 129-147, 245-261, 1878.

Ida, S. and K. Nakazawa, Collisional probability of planetesimals revolving in the solar gravitational field. III, Astron. Astrophys., 224, 303-315, 1989.

Ida, S. and J. Makino, $N$-body simulation of gravitational interaction between planetesimals and a protoplanet. I-Velocity distribution of planetesimals, Icarus, 96, 107-120, 1992a.

Ida, S. and J. Makino, $N$-body simulation of gravitational interaction between planetesimals and a protoplanet. II-Dynamical friction, Icarus, 98, 28-37, 1992b.

Ida, S., R. M. Canup, and G. R. Stewart, Lunar accretion from an impactgenerated disk, Nature, 389, 353-357, 1997.

Kokubo, E. and S. Ida, On runaway growth of planetesimals, Icarus, 123, 180-191, 1996.

Kokubo, E. and S. Ida, Oligarchic growth of protoplanets, Icarus, 131, 171-178, 1998.

Lissauer, J. J., A. F. Berman, Y. Greenzweig, and D. M. Kary, Accretion of mass and spin angular momentum by a planet on an eccentric orbit, Icarus, 127, 65-92, 1997.

Lucy, L. B., A numerical approach to the testing of the fission hypothesis, Astron. J., 82, 1013-1024, 1977.

Mizuno, H. and A. P. Boss, Tidal disruption of dissipative planetesimals, Icarus, 63, 109-133, 1985.
Monaghan, J. J., Smoothed particle hydrodynamics, Ann. Rev. Astron. Astrophys., 30, 543-574, 1992.

Monaghan, J. J. and J. C. Lattanzio, A refined particle method for astrophysical problems, Astron. Astrophys., 149, 135-143, 1985.

Nakazawa, K. and S. Ida, Hill's approximation in the three-body problem, Prog. Theor. Phys. Suppl., 96, 167-174, 1988.

Nakazawa, K., S. Ida, and Y. Nakagawa, Collisional probability of planetesimals revolving in the solar gravitational field. I-Basic formulation, Astron. Astrophys., 220, 293-300, 1989a.

Nakazawa, K., S. Ida, and Y. Nakagawa, Collisional probability of planetesimals revolving in the solar gravitational field. II-The validity of the two-body approximation, Astron. Astrophys., 221, 342-347, 1989 b.

Safronov, V. S. and E. V. Zvjagina, Relative sizes of the largest bodies during the accumulation of planets, Icarus, 10, 109-115, 1969.

Tillotson, J. H., Metallic equations of state for hypervelocity impact, Rep. GA-3216, July 18, Gen At., San Diego, CA, 1962.

Wada, K., E. Kokubo, and J. Makino, High-resolution simulations of a Moon-forming impact and postimpact evolution, Astrophys. J., 638, 1180-1186, 2006.

Watanabe, S.-I. and S. M. Miyama, Collision and tidal interaction between planetesimals, Astrophys. J., 391, 318-335, 1992.

Wood, J. A., Moon over Mauna Loa-A review of hypotheses of formation of earth's moon, in Origin of the Moon, edited by W. K. Hartmann, R. J. Phillips, and G. J. Taylor, 781 pp., Lunar and Planetary Institute, Houston, 1986.

A. Nouda (e-mail: anouda@geo.titech.ac.jp), H. Emori, and K. Nakazawa 